\title{
A geometric look at MHD and the Braginsky dynamo
}

\author{
Andrew D. Gilbert ${ }^{1} \&$ Jacques Vanneste ${ }^{2}$ \\ ${ }^{1}$ Department of Mathematics, College of Engineering, Mathematics and Physical Sciences, University of \\ Exeter, Exeter EX4 4QF, UK \\ ${ }^{2}$ School of Mathematics and Maxwell Institute for Mathematical Sciences, University of Edinburgh, \\ Edinburgh EH9 3FD, UK
}

October 20, 2020

\begin{abstract}
This paper considers magnetohydrodynamics (MHD) and some of its applications from the perspective of differential geometry, considering the dynamics of an ideal fluid flow and magnetic field on a general three-dimensional manifold, equipped with a metric and an induced volume form. The benefit of this level of abstraction is that it clarifies basic aspects of fluid dynamics such as how certain quantities are transported, how they transform under the action of mappings (for example the flow map between Lagrangian labels and Eulerian positions), how conservation laws arise, and the origin of certain approximations that preserve the mathematical structure of classical mechanics.

First, the governing equations for ideal MHD are derived in a general setting by means of an action principle, and making use of Lie derivatives. The way in which these equations transform under a pull back, by the map taking the position of a fluid parcel to a background location, is detailed. This is then used to parameterise Alfvén waves using concepts of pseudomomentum and pseudofield, in parallel with the development of Generalised Lagrangian Mean theory in hydrodynamics. Finally non-ideal MHD is considered with a sketch of the development of the Braginsky $\alpha \omega$-dynamo in a general setting. Expressions for the $\alpha$-tensor are obtained, including a novel geometric formulation in terms of connection coefficients, and related to formulae found elsewhere in the literature.
\end{abstract}

\section{Introduction}

In this paper we discuss some aspects of magnetohydrodynamics (MHD) from a geometric perspective: we consider an ideal fluid flow and magnetic field occupying a three-dimensional manifold $\mathcal{M}$ equipped with a metric $g$ and induced volume form $\mu$. We discuss several topics where this level of abstraction is useful in understanding mathematical structure, approximations and phenomena, even when we may ultimately be operating in everyday three-dimensional space. Taking a geometric perspective on fluid dynamics was pioneered in the seminal paper Arnold (1966); this and related works are reviewed in the book Arnold and Khesin (1998). Building on the mathematical foundations of classical mechanics, Arnold showed that an incompressible ideal fluid flow may be considered as a geodesic on a manifold known as $\operatorname{SDiff}(\mathcal{M})$, the space of all volume-preserving diffeomorphisms of the space $\mathcal{M}$, and that this space has an underlying Lie group structure. Here $\operatorname{SDiff}(\mathcal{M})$ has a metric that comes from the metric $g$ on $\mathcal{M}$, and this is needed to define geodesics: the shortest path taken by a point in this big space $\operatorname{SDiff}(\mathcal{M})$ gives the flow map, say $\phi_{t}$, that moves all the Lagrangian fluid parcels for the actual flow in $\mathcal{M}$. Arnold's geometric perspective on a classical topic led to many new and varied results in theoretical fluid dynamics, for example in stability theory (e.g. Holm et al., 1985), in Hamiltonian mechanics, understanding the origin of conservation laws and determining systematic approximate or reduced fluid systems (e.g. Morrison, 1982; McIntyre and Shepherd, 1987; Salmon, 1988; Shepherd, 1990; Morrison, 1998; Webb, 2018), and in modelling fluctuations and waves in laminar and turbulent flows (e.g. Foias, Holm and Titi, 2001; Soward and Roberts, 
2010; Gilbert and Vanneste, 2018). Often the key advantage of a geometric perspective is that the transformations or approximations used naturally preserve the underlying geometry of fluid motion, in other words the essential structure of classical mechanics, together with its conservation laws. A modern geometric approach also underlies mimetic numerical methods (e.g. Qin. Guan and Tang, 2009; Leok and Shingel, 2012; Thuburn and Cotter, 2015; Kraus, Tassic and Grassod, 2016; Palha and Gerritsma, 2017), which are designed to respect key mathematical properties; an example is that a discrete approximation to vector calculus operators should preserve the fundamental identity that $\operatorname{div} \operatorname{curl} \boldsymbol{f}=0$, or in a more general setting that $\mathrm{d}^{2} \gamma=0$ for the exterior derivative $\mathrm{d}$ of a differential form $\gamma$. Failing to preserve such identities easily leads to spurious physical effects whose origin is purely numerical.

While there has been more study of geometric methods applied to hydrodynamics than to MHD (reviewed in the book Webb, 2018), many key ideas in fact arose in parallel with (or following) similar developments in the latter field. One notion familiar to anyone dealing with a magnetic field $\boldsymbol{b}$ (or vorticity $\boldsymbol{\omega}$ ) is that ideal transport (that is, with zero diffusion) in incompressible flow is achieved by means of the Cauchy solution. This relates a field vector $\boldsymbol{b}(\boldsymbol{x})$ attached to a Lagrangian fluid parcel at $\boldsymbol{x}$ that is transported to a point $\boldsymbol{x}^{\prime}$, to the new vector $\boldsymbol{b}^{\prime}\left(\boldsymbol{x}^{\prime}\right)$ with components

$$
b_{i}^{\prime}\left(\boldsymbol{x}^{\prime}\right)=J_{i j}(\boldsymbol{x}) b_{j}(\boldsymbol{x}), \quad \text { where } J_{i j}=\partial x_{i}^{\prime} / \partial x_{j}
$$

is the Jacobian matrix $J$ of the transformation $\psi: \boldsymbol{x} \mapsto \boldsymbol{x}^{\prime}$.

In geometric parlance this is the push forward of the field under the map $\psi$, and is a standard way of looking at the transport of magnetic field in ideal or near-ideal MHD or dynamo theory. What is less intuitive perhaps, is that some quantities are not transported in this way, as a (contravariant) vector field, but as a 1-form field (or covariant vector field), an example of this being momentum. In a key paper, Soward (1972) showed that the natural way of tranporting momentum $\boldsymbol{p}$ from place to place is using the inverse transpose of the same Jacobian,

$$
p_{i}^{\prime}\left(\boldsymbol{x}^{\prime}\right)=K_{i j}(\boldsymbol{x}) p_{j}(\boldsymbol{x}), \quad K=\left(J^{-1}\right)^{\mathrm{T}} .
$$

In the language of differential geometry this is a push forward of the momentum $\boldsymbol{p}$ considered as a 1-form, under the map $\psi$.

Thus, there are two types of transport relevant to everyday vector fields in incompressible flow, that given by (1.1) and that given by (1.2). If we have quantities transported in this way then the inner product $\boldsymbol{b} \cdot \boldsymbol{p}$ is conserved. As an example, if the map $\psi$ is the flow map $\phi_{t}$, moving Lagrangian parcels from their positions at time $t=0$ to those at time $t$, then magnetic field $\boldsymbol{b}$ and vorticity $\boldsymbol{\omega}$ evolve according to (1.1), and a scalar gradient $\boldsymbol{\nabla} \chi$ and (in a suitable gauge) the magnetic vector potential $\boldsymbol{a}$ evolve according to (1.2). We then have immediately that $\boldsymbol{\omega} \cdot \boldsymbol{\nabla} \chi$ and $\boldsymbol{b} \cdot \boldsymbol{a}$ are conserved on fluid parcels. These are respectively Ertel's potential vorticity (Ertel, 1942) and the magnetic helicity density (see Moffatt and Dormy, 2019).

The picture is more complicated for the momentum $\boldsymbol{p}$ because this can be redistributed by the pressure field, so that the transport of momentum does not occur via (1.2) under the flow map $\phi_{t}$. However this equation does give the means of moving momentum from point to point in any kind of Lagrangian averaging. This was realised in Soward's (1972) paper, which built on earlier work of Frieman and Rotenberg (1960) and Eckart (1963), and was developed as Generalised Lagrangian Mean (GLM) theory in the papers Andrews and McIntyre (1978a, b) ; see the book Bühler (2009) for further developments. The key idea here is that it is often beneficial to take averages of fluid dynamical equations not at a fixed location in a family of flow realisations - an Eulerian average - but to take a Lagrangian average, over the locations of a single Lagrangian parcel. In this case it is necessary to move vectors from place to place in the flow, and the transformation rules (1.1) and (1.2) come into play. If quantities are moved in this way (rather than parallel transport, say), remarkable properties of the resulting Lagrangian averages emerge; in particular the structure of the Euler equation is preserved to the extent that Kelvin's circulation theorem continues to apply; for recent developments see Holm (2002b), Soward and Roberts (2010), Gilbert and Vanneste (2018) and the book Webb (2018).

Although all this machinery works beautifully and is well established in the literature, together with applications, we argue that it is only by stepping back and considering some of these fluid dynamical 
systems in a more abstract setting that it is clear why these methods work, what is the origin of various transformation and conservation laws, and where choices can and cannot be made. For example working in Euclidean space $\mathbb{R}^{3}$ with Cartesian coordinates $(x, y, z)$ and metric $g=\mathrm{d} x^{2}+\mathrm{d} y^{2}+\mathrm{d} z^{2}$, it is too easy to switch between vector fields such as the velocity $\boldsymbol{u}$ and 1-form fields such as the momentum $\boldsymbol{p}$, which have the same components (up to the factor of density $\rho$ ) even though they are very different objects in terms of their properties under transport. Working on a general manifold $\mathcal{M}$ with a metric $g$ and an induced volume form $\mu$ forces one to establish what type of object one is dealing with at the outset, and with this, theory can become easier and clearer, drawing on well-established results in differential geometry. The advantage of the use of differential geometry and particularly Lie derivatives has been stressed by several authors in MHD, including Tur and Yanovskv (1993), Marsden and Shkoller (2001), Holm (2002b), Roberts and Soward (2006a, b), Arter (2013a,b), Soward and Roberts (2014) and Webb (2018). Even working in $\mathbb{R}^{3}$, the use of a formulation based on a general metric $g$ can avoid complications switching from Cartesian coordinates to, say, cylindrical polars, and is well-nigh essential if non-orthogonal coordinates are employed, for example when a coordinate labels surfaces of constant buoyancy in a geophysical fluid dynamical setting (Gilbert and Vanneste, 2020b).

In the present paper, we discuss three related topics in which we place MHD in an abstract geometric setting. In section 2 we derive the MHD equations from an action principle: this starting point is useful as it forces us to identify the different types of objects that can be used to describe the flow field (velocity $u$, momentum $\rho \nu$ ) and magnetic field (flux 2-form $B$, vector field $b$ and 1-form field $\beta$ ), how they are related using the metric and volume form, and how they appear in the equations of motion. Compressible fluid flow is allowed, and the magnetic pressure term emerges in the calculation. The section is standard material but takes a geometric approach to recast the classical derivation of Newcomb (1962), reviewed in Van Kampen and Felderhoff (1967), Morrison (1998) and Webb (2018). We also discuss the conservation of different types of helicity within the geometical framework (Arnold and Khesin, 1998; Fukumoto, 2008; Webb et al., 2014a b). With the equations established in geometric form, in section 3 we discuss how they transform under a pull back (or push forward) by a mapping. This gives rise to the notion of pseudomomentum and pseudo (magnetic) field, and these are calculated for finite amplitude Alfvén waves on a uniform magnetic field threading an incompressible fluid (Alfvén and Falthammar, 1950). We also consider cross helicity under Lagrangian averaging (Holm, 2002a).

In section 4 we discuss transformation of the induction equation under a mapping and sketch its use in the Braginsky (1964a, b) dynamo: here one starts with a background flow that is not a dynamo but gives an $\omega$-effect, stretching out field that is transverse to streamlines. Then waves are included by means of a Lagrangian map: through the diffusion term, these can generate an $\alpha$-effect and close the dynamo cycle to give an $\alpha \omega$-dynamo. We note that the use of mappings and transport of field is well-established following Soward (1972), with further developments in Roberts and Soward (2006a b) and a comprehensive treatment given recently in Soward and Roberts (2014). Although these papers use many concepts from differential geometry implicitly, the goal of the present paper is to make the machinery explicit, and so both simplify and unify previous methods and results: to our knowledge the perspective that we aim to convey is novel and we also obtain a new description of the $\alpha$-effect in terms of a connection tensor. The present paper is self-contained, but the framework follows on from Gilbert and Vanneste (2018); a companion paper Gilbert and Vanneste (2020a) studies how fluid systems can be written in a conservation form, including MHD and shallow water MHD. 


\section{Ideal MHD from an action principle}

\subsection{Usual MHD equations}

For reference purposes, we begin by setting out the equations for MHD in usual Euclidean space $\mathbb{R}^{3}$ using standard notation, namely,

$$
\begin{aligned}
\rho\left(\partial_{t} \boldsymbol{u}\right. & \left.+\boldsymbol{\omega} \times \boldsymbol{u}+\frac{1}{2} \boldsymbol{\nabla}|\boldsymbol{u}|^{2}\right)+\nabla p=\boldsymbol{j} \times \boldsymbol{b}, \\
\partial_{t} \boldsymbol{b} & =\boldsymbol{\nabla} \times(\boldsymbol{u} \times \boldsymbol{b})-\eta \boldsymbol{\nabla} \times \boldsymbol{j}, \\
\boldsymbol{\omega} & =\boldsymbol{\nabla} \times \boldsymbol{u}, \quad \boldsymbol{j}=\boldsymbol{\nabla} \times \boldsymbol{b}, \quad \boldsymbol{\nabla} \cdot \boldsymbol{b}=0 .
\end{aligned}
$$

Here we have allowed the field to be non-ideal, with (constant) magnetic diffusivity $\eta$ : sections 2 and 3 will consider ideal MHD, $\eta=0$, and section 4 dynamos with $\eta>0$. We have not introduced viscosity; see Gilbert. Riedinger and Thuburn (2014) and Gilbert and Vanneste (2020a) for discussion of how the divergence of a stress tensor is taken in a geometric setting. For convenience the magnetic permeability $\mu_{0}$ is scaled out, the true magnetic field is in fact $\mu_{0}^{1 / 2} \boldsymbol{b}$. We allow either incompressible flow with $\boldsymbol{\nabla} \cdot \boldsymbol{u}=0$ or ideal compressible flow, in which case an equation of state $p=p(\rho, s)$ is also needed, with $s$ as the entropy and $\rho$ the density governed by

$$
\begin{gathered}
\partial_{t} s+\boldsymbol{u} \cdot \boldsymbol{\nabla} s=0, \\
\partial_{t} \rho+\boldsymbol{\nabla} \cdot(\rho \boldsymbol{u})=0 .
\end{gathered}
$$

\subsection{Geometric setting and transport}

Having set out the equations in ordinary three-dimensional space, we now place these in an abstract setting, and will use lighter face quantities for all the various physical fields to stress this. Our fluid domain is now an orientable three-dimensional manifold $\mathcal{M}$, with or without a boundary $\partial \mathcal{M}$; examples include all of $\mathbb{R}^{3}$ or a solid sphere. For simplicity we will avoid discussion of manifolds with 'holes' in them, for example a spherical shell; in other words we restrict to manifolds $\mathcal{M}$ where any curve or closed surface can be contracted to a point. In ideal fluid mechanics any flow or magnetic vector field is taken parallel to the boundary, $u, b \| \partial \mathcal{M}$.

We assume that the reader has knowledge of the fundamentals of differential geometry, as given by, for example, Hawking and Ellis (1973), Schutz (1980), Frankel (1997) or Besse and Frisch (2017). In particular we make use of vectors, differential forms, the Lie derivative $\mathcal{L}$, inner product $\lrcorner$, exterior derivative $\mathrm{d}$, the Hodge star operator $\star$, and the musical raising and lowering operators $\sharp$ and $b$. To discuss fluid dynamics we need $\mathcal{M}$ to be equipped with a metric $g$ and induced volume form $\mu$, and occasionally it is useful to refer to the corresponding covariant derivative $\nabla$. We make frequent use, often without comment, of Cartan's formula: for any differential form $\gamma$,

$$
\left.\left.\mathcal{L}_{u} \gamma=\mathrm{d}(u\lrcorner \gamma\right)+u\right\lrcorner \mathrm{d} \gamma .
$$

Our first concern is the magnetic field, whose most fundamental property perhaps is the absence of magnetic monopoles, so that the integral of magnetic flux over any closed surface $\mathcal{S}$ vanishes. From the geometric viewpoint this means that the magnetic field is most naturally represented by the magnetic flux 2-form $B$ (Tur and Yanovsky, 1993; Frankel, 1997), which is required to be closed, $\mathrm{d} B=0$. Then, as any closed surface $\mathcal{S}$ in $\mathcal{M}$ bounds a volume $\mathcal{V}$ with $\mathcal{S}=\partial \mathcal{V}$, we have

$$
\int_{\mathcal{S}} B=\int_{\mathcal{V}} \mathrm{d} B=0
$$

using the generalised Stokes theorem (corresponding to the usual divergence theorem in this instance). The focus on the magnetic flux $B$ is natural in a geometric setting since 2-form fields are naturally integrated over surfaces. In ideal MHD, magnetic field is transported in the fluid flow, as per Alfvén's theorem, and in the general setting this corresponds to requiring that $B$ be Lie dragged in the fluid flow (Tur and Yanovskv, 1993),

$$
\partial_{t} B+\mathcal{L}_{u} B=0
$$


This preserves the condition $\mathrm{d} B=0$ since $\mathrm{d}$ commutes with Lie and time derivatives.

We are often more used to thinking of magnetic field as a magnetic vector field $b$ rather than a 2 -form field $B$, and $b$ is easily defined using the volume 3-form via $b\lrcorner \mu=B$. In this case it follows from (2.8) that $b$ is transported according to

$$
\partial_{t} b+\mathcal{L}_{u} b+b \operatorname{div} u=0
$$

where $\operatorname{div} u$ is the divergence of the flow field $u$ given by

$$
\left.\mathcal{L}_{u} \mu=\mathrm{d}(u\lrcorner \mu\right)=\mu \operatorname{div} u .
$$

This transport of $b$ takes into account the action of compressible flow on the field through the $b \operatorname{div} u$ term, and for this reason $b$ is sometimes referred to as a 'tensor of weight -1 ' (Roberts and Soward, 2006a). The solenoidal property of $b$, namely $\operatorname{div} b=0$, follows from $\mathrm{d} B=0$.

Other transported quantities include the entropy $s$ which is a scalar field obeying

$$
\partial_{t} s+\mathcal{L}_{u} s=0
$$

and density $\rho$. Again, more fundamental than density itself is the mass 3-form $m$, in that integration of $m$ over a volume gives the mass contained therein. The mass form is again Lie dragged via

$$
\partial_{t} m+\mathcal{L}_{u} m=0,
$$

giving mass conservation, while the density $\rho$, now defined by $m=\rho \mu$, is a scalar of weight -1 obeying

$$
\partial_{t} \rho+\mathcal{L}_{u} \rho+\rho \operatorname{div} u=0 .
$$

In short, in an ideal setting the fundamental quantities of magnetic flux $B$, entropy $s$ and mass $m$ are all Lie dragged in the flow field in the same way via (2.8), (2.11) and (2.12), while the derived quantities of magnetic vector field $b$ and density $\rho$ obey the slightly more complex equations (2.9) and (2.13), bringing in the divergence of the flow field $u$. This latter effect can also be absorbed by dividing $b$ by $\rho$; see Arter (2013a) for a development along these lines, and see Tur and Yanovskv (1993) for discussion of Lie-dragging and how ideal invariants are systematically formulated within this framework.

\subsection{Ideal MHD from an action principle}

We now derive the equations for the flow $u$ starting with an action principle (Newcomb, 1962; Morrison, 1998; Webb, 2018). We follow the geometrical discussion in Gilbert and Vanneste (2018) with an additional magnetic field; see Holm et al. (1998) for an equivalent derivation in the more general framework of EulerPoincaré systems, and Arnold and Khesin (1998) for the treatment of incompressibility case. For this we need the Lagrangian flow map $\phi \equiv \phi_{t}$ (we drop the subscript $t$ ) a time-dependent diffeomorphism of $\mathcal{M}$ which takes fluid elements from their positions $x$ at time $t=0$ to their current position $\phi(x, t)$. This map is assumed to be invertible and differentiable as much as is needed. In terms of $\phi$ the flow $u$, which is a time-dependent vector field on $\mathcal{M}$, is given by

$$
u=\dot{\phi} \circ \phi^{-1} .
$$

The kinetic energy of the fluid may then be expressed in terms of an integral over the initial particle locations (i.e. at $t=0$ ), used as Lagrangian labels, by

$$
\mathcal{K}=\int_{\mathcal{M}} \frac{1}{2} g(\dot{\phi}, \dot{\phi}) m_{0}
$$

where $m_{0}=\rho_{0} \mu$ is the initial mass distribution, or in terms of the current positions and current mass distribution $m$ (i.e. at a general time $t$ ) by

$$
\mathcal{K}=\int_{\mathcal{M}} \frac{1}{2} g(u, u) m .
$$


The full action, in terms of current positions, is then

$$
\mathcal{A}[\phi]=\int \mathrm{d} t \int_{\mathcal{M}}\left[\frac{1}{2} g(u, u) m-\rho e(\rho, s) \mu-\frac{1}{2} g(b, b) \mu\right] .
$$

Here the fields $m, s, B, \rho$ and $b$ depend on the flow map since they are obtained from their initial values by the push forwards,

$$
m=\phi_{*} m_{0}, \quad s=\phi_{*} s_{0}, \quad B=\phi_{*} B_{0},
$$

and as above $m=\rho \mu$ and $B=b\lrcorner \mu$. For magnetic field this corresponds to the Cauchy solution. In the action, $e(\rho, s)$ is the internal energy per unit mass, and it is notable that this and the kinetic energy are weighted with mass $m=\rho \mu$, whereas the magnetic energy involves the vector field $b$ rather than the flux 2 -form $B$, and is weighted with volume $\mu$.

Using Hamilton's principle, we require that the action is stationary under variations in the paths of the fluid particles over some time interval. We achieve this in the present framework by replacing the flow map $\phi$ in the above by the perturbed flow map $\phi_{\varepsilon}=\psi_{\varepsilon} \circ \phi$. Here $\psi_{\varepsilon}$ is a family of diffeomorphisms of $\mathcal{M}$ dependent on time and on a scalar parameter $\varepsilon$, equal to the identity for $\varepsilon=0$. We require that the action be stationary with respect to such variations,

$$
\left.\frac{\mathrm{d}}{\mathrm{d} \varepsilon}\right|_{\varepsilon=0} \mathcal{A}\left[\phi_{\varepsilon}\right]=0
$$

If we fix time $t$ and vary $\varepsilon$ around $\varepsilon=0$ the family of maps $\psi_{\varepsilon}$ gives a vector field $w$, formally defined by

$$
w=\left.\frac{\mathrm{d} \psi_{\varepsilon}}{\mathrm{d} \varepsilon} \circ \psi_{\varepsilon}^{-1}\right|_{\varepsilon=0},
$$

and this field is parallel to the boundary of $\mathcal{M}$. On the other hand if we fix $\varepsilon$ and vary $t$ we obtain the flow velocity under $\phi_{\varepsilon}$ as in (2.14) with

$$
u_{\varepsilon}=\dot{\phi}_{\varepsilon} \circ \phi_{\varepsilon}^{-1} .
$$

Key is the relationship between $u_{\varepsilon}$ and $w$, how a variation in the map affects the resulting particle velocities, and this is

$$
\left.\frac{\mathrm{d}}{\mathrm{d} \varepsilon}\right|_{\varepsilon=0} u_{\varepsilon}=\partial_{t} w+\mathcal{L}_{u} w=\partial_{t} w-\mathcal{L}_{w} u
$$

This key identity is easily shown using coordinates (or more abstractly in Gilbert and Vanneste (2018), appendix B) by writing from (2.20, 2.21)

$$
u_{\varepsilon}\left(\phi_{\varepsilon}(x, t), t\right)=\partial_{t} \phi_{\varepsilon}(x, t), \quad w_{\varepsilon}\left(\phi_{\varepsilon}(x, t), t\right)=\partial_{\varepsilon} \phi_{\varepsilon}(x, t),
$$

and equating $\partial_{t} \partial_{\varepsilon} \phi_{\varepsilon}=\partial_{\varepsilon} \partial_{t} \phi_{\varepsilon}$ to give

$$
\partial_{\varepsilon} u_{\varepsilon}^{i}+\left(\partial_{j} u_{\varepsilon}^{i}\right)\left(\partial_{\varepsilon} \phi_{\varepsilon}^{j}\right)=\partial_{t} w_{\varepsilon}^{i}+\left(\partial_{j} w_{\varepsilon}^{i}\right)\left(\partial_{t} \phi_{\varepsilon}^{j}\right)
$$

or

$$
\partial_{\varepsilon} u_{\varepsilon}^{i}+\left(\partial_{j} u_{\varepsilon}^{i}\right) w_{\varepsilon}^{j}=\partial_{t} w_{\varepsilon}^{i}+\left(\partial_{j} w_{\varepsilon}^{i}\right) u_{\varepsilon}^{j},
$$

which, evaluated at $\varepsilon=0$, amounts to (2.22).

For other quantities in the action, we have from the earlier transport equations (2.8 2.13) that

$$
\begin{aligned}
& \left.\frac{\mathrm{d}}{\mathrm{d} \varepsilon}\right|_{\varepsilon=0} m_{\varepsilon}=-\mathcal{L}_{w} m=-\mathcal{L}_{w}(\rho \mu)=-\operatorname{div}(\rho w) \mu, \\
& \left.\frac{\mathrm{d}}{\mathrm{d} \varepsilon}\right|_{\varepsilon=0} b_{\varepsilon}=-\mathcal{L}_{w} b-(\operatorname{div} w) b, \\
& \left.\frac{\mathrm{d}}{\mathrm{d} \varepsilon}\right|_{\varepsilon=0} s_{\varepsilon}=-\mathcal{L}_{w} s, \\
& \left.\frac{\mathrm{d}}{\mathrm{d} \varepsilon}\right|_{\varepsilon=0} \rho_{\varepsilon}=-\operatorname{div}(\rho w),
\end{aligned}
$$


where the $\varepsilon$ subscript denotes their values under transport by $\phi_{\varepsilon}$ from the same initial conditions in (2.18).

The requirement (2.19) that the action $\mathcal{A}\left[\phi_{\varepsilon}\right]$ be stationary at $\varepsilon=0$ becomes

$$
\begin{aligned}
\int \mathrm{d} t \int_{\mathrm{M}}\left[g\left(u, \partial_{t} w+\mathcal{L}_{u} w\right) m\right. & -\frac{1}{2} g(u, u) \mathcal{L}_{w} m+(\rho e)_{\rho} \operatorname{div}(\rho w) \mu+\rho e_{s}\left(\mathcal{L}_{w} s\right) \mu \\
& \left.+g\left(b, \mathcal{L}_{w} b+(\operatorname{div} w) b\right) \mu\right]=0 .
\end{aligned}
$$

We need to pull out the arbitrary vector field $w$ from this. First we take $w$ to be identically zero outside some time interval, which we do not need to give explicitly, so we can apply integration by parts with respect to time. Secondly we can use integration by parts in space. Let $\gamma$ be any 3-form, then for any vector field parallel to the boundary of $\mathcal{M}$ (such as $u, w, b)$ we have

$$
\left.\left.\int_{\mathcal{M}} \mathcal{L}_{u} \gamma=\int_{\mathcal{M}} \mathrm{d}(u\lrcorner \gamma\right)=\int_{\partial \mathcal{M}} u\right\lrcorner \gamma=0 .
$$

Together with the Leibniz rule for Lie derivatives, this means that we can integrate by parts and shift the Lie derivative from a term in a product to the remaining terms, while introducing a minus sign.

Using these and similar rules we can replace quantities in the above integral by their equivalents. To keep notation light we denote this by $\simeq$. We define the key quantities, the 1 -forms,

$$
\nu=g(u, \cdot)=u_{b},
$$

and

$$
\beta=g(b, \cdot)=b_{b}=\star B,
$$

where $\star$ is the Hodge star operator. We then have for the kinetic energy terms,

$$
\begin{aligned}
g\left(u, \partial_{t} w\right) m & \left.\left.\left.=\mu \rho \nu\lrcorner \partial_{t} w \simeq-w\right\lrcorner \partial_{t}(\rho \nu) \mu=-w\right\lrcorner \nu\left(\partial_{t} \rho\right) \mu-w\right\lrcorner\left(\partial_{t} \nu\right) \rho \mu, \\
g\left(u, \mathcal{L}_{u} w\right) m & \left.\left.=\mu \rho \nu\lrcorner \mathcal{L}_{u} w \simeq-w\right\lrcorner \mathcal{L}_{u}(\rho \nu) \mu-\rho w\right\lrcorner \nu \mathcal{L}_{u} \mu \\
& \left.\left.\left.=-w\lrcorner \mathcal{L}_{u}(\rho \nu) \mu-\rho w\right\lrcorner \nu(\operatorname{div} u) \mu=-w\right\lrcorner\left(\mathcal{L}_{u} \nu\right) \rho \mu+w\right\lrcorner \nu\left(\partial_{t} \rho\right) \mu, \\
-\frac{1}{2} g(u, u) \mathcal{L}_{w} m & \left.\left.\simeq m \mathcal{L}_{w} \frac{1}{2} g(u, u)=m w\right\lrcorner \frac{1}{2} \mathrm{~d} g(u, u)=w\right\lrcorner \frac{1}{2} \rho \operatorname{d} g(u, u) \mu,
\end{aligned}
$$

using (2.13) for (2.35). For the internal energy terms we have

$$
\begin{aligned}
(\rho e)_{\rho} \operatorname{div}(\rho w) \mu+\rho e_{s}\left(\mathcal{L}_{w} s\right) \mu & =(\rho e)_{\rho} \mathcal{L}_{w}(\rho \mu)+\rho e_{s}\left(\mathcal{L}_{w} s\right) \mu \\
& \simeq-\mathcal{L}_{w}\left[(\rho e)_{\rho}\right] \rho \mu+\rho e_{s}\left(\mathcal{L}_{w} s\right) \mu \\
& \left.=-w\lrcorner \mathrm{~d}\left[(\rho e)_{\rho}\right] \rho \mu+w\right\lrcorner\left(\rho e_{s} \mathrm{~d} s\right) \mu \\
& =w\lrcorner(-\rho \mathrm{d} h+\rho T \mathrm{~d} s) \mu \\
& =-w\lrcorner d p \mu,
\end{aligned}
$$

where we have used thermodynamic relations for pressure $p$, temperature $T$ and enthalpy $h$,

$$
h=(\rho e)_{\rho}=e+p / \rho, \quad T=e_{s}, \quad \mathrm{~d} h=\rho^{-1} \mathrm{~d} p+T \mathrm{~d} s .
$$

Finally, for the magnetic terms we have

$$
\begin{aligned}
& \left.\left.\left.\left.g\left(b, \mathcal{L}_{w} b\right) \mu=-\mu \beta\right\lrcorner \mathcal{L}_{b} w \simeq w\right\lrcorner\left(\mathcal{L}_{b} \beta\right) \mu+w\right\lrcorner \beta \mathcal{L}_{b} \mu=w\right\lrcorner\left(\mathcal{L}_{b} \beta\right) \mu, \\
& \left.g(b,(\operatorname{div} w) b) \mu=g(b, b) \mathcal{L}_{w} \mu \simeq-\mathcal{L}_{w}[g(b, b)] \mu=-w\right\lrcorner \mathrm{d} g(b, b) \mu,
\end{aligned}
$$

using that $\left.\mathcal{L}_{b} \mu=\mathrm{d}(b\lrcorner \mu\right)=\mathrm{d} B=0$. These expressions are inserted into (2.30) which must hold for arbitrary $w$, and from this we obtain the momentum equation in the form

$$
\rho\left[\partial_{t} \nu+\mathcal{L}_{u} \nu-\frac{1}{2} \mathrm{~d} g(u, u)\right]+\mathrm{d} p=\mathcal{L}_{b} \beta-\mathrm{d} g(b, b),
$$

for ideal MHD flow in a general setting. 


\subsection{Discussion}

Several remarks are in order. First, using Cartan's formula (2.6) and noting that $g(u, u)=\nu\lrcorner u, g(b, b)=b\lrcorner \beta$, (2.41) may be expressed as

$$
\left.\left.\rho\left[\partial_{t} \nu+u\right\lrcorner \mathrm{d} \nu+\frac{1}{2} \mathrm{~d} g(u, u)\right]+\mathrm{d} p=b\right\lrcorner \mathrm{d} \beta,
$$

which is equivalent to (2.1) where we identify

$$
\zeta=\mathrm{d} \nu \quad \text { and } \quad j=\mathrm{d} \beta
$$

as the vorticity and current 2-forms. Both of these can be converted to vector fields in the same way that $b\lrcorner \mu=B$ relates the magnetic flux 2-form $B$ to the corresponding vector field $b$. Likewise equation (2.8) for evolution of magnetic field may be rewritten as

$$
\left.\partial_{t} B+\mathrm{d}(u\lrcorner B\right)=0
$$

which is analogous to (2.2) for $\eta=0$, with the exterior derivative playing the role of the curl.

Secondly, in the momentum equation we have found that terms emerge for the transport, not of the velocity vector $u$, but for the corresponding 1-form momentum $\nu=u_{b}$ (strictly the momentum is $\rho \nu$ ). Although these two are very similar, and in relativity would more-or-less be identified, in the context of geometrical fluid dynamics it is important to keep the distinction between the two quantities. Likewise in determining the Lorentz force term we are driven to introduce the 1 -form $\beta$. Both $b$ and $\beta$ are related back to the fundamental magnetic flux 2-form $B$ since $b\lrcorner \mu=B$ and $\beta=b_{\mathrm{b}}$, but it is convenient to distinguish them using different symbols. It is not clear that there is a good name for the magnetic 1-form field $\beta$ (we have the magnetic vector field $b$ and the magnetic flux 2-form B) but the distinction between the two quantities $b$ and $\beta$ and their properties has been stressed in Holm (2002a) and Roberts and Soward (2006a), for reasons that become apparent in section 3 .

Thirdly, note that a vorticity equation can be obtained in terms of Lie derivatives (Arter, 2013a) by dividing (2.41) by $\rho$ to give

$$
\left.\partial_{t} \nu+\mathcal{L}_{u} \nu-\frac{1}{2} \mathrm{~d} g(u, u)+\rho^{-1} \mathrm{~d} p=\rho^{-1} b\right\lrcorner \mathrm{d} \beta
$$

then applying $d$ to obtain

$$
\left.\partial_{t} \zeta+\mathcal{L}_{u} \zeta-\rho^{-2} \mathrm{~d} \rho \wedge \mathrm{d} p=\mathrm{d}\left(\rho^{-1} b\right\lrcorner j\right)=\mathcal{L}_{b / \rho} j
$$

using (2.43) and Cartan's formula (2.6). The quantity $\zeta$ is referred to as the potential vorticity by Arter (2013a). This should not be confused with the (Rossby-Ertel) potential vorticity 3 -form $\zeta \wedge \mathrm{d} s$, or the potential vorticity scalar $Q$ defined by $\rho Q \mu=\zeta \wedge \mathrm{d} s$, both of which are Lie transported by $u$ in the absence of magnetic field.

Perhaps a more familiar route for some readers is to express the momentum and induction equations in terms of the covariant derivative. Here we write the components of $u$ as $u^{i}$ and those of $\nu$ as $u_{i}$, etc. Using standard results, in particular that

$$
\mathcal{L}_{u} \nu=\left(\nabla_{u} u\right)_{b}+\frac{1}{2} \mathrm{~d} g(u, u)
$$

(Arnold and Khesin, 1998), the momentum equation (2.41) may be written as

$$
\rho\left(\partial_{t} u_{i}+u^{j} \nabla_{j} u_{i}\right)+\nabla_{i}\left(p+\frac{1}{2} g_{j k} b^{j} b^{k}\right)=b^{j} \nabla_{j} b_{i}
$$

In this formulation it is interesting to see the magnetic pressure term $\frac{1}{2} g_{j k} b^{j} b^{k}$ emerge, with no corresponding term involving $\frac{1}{2} g_{j k} u^{j} u^{k}$. For magnetic field, equation (2.9) can be reexpressed as a familiar form of the induction equation,

$$
\partial_{t} b^{i}+u^{j} \nabla_{j} b^{i}-b^{j} \nabla_{j} u^{i}+\left(\nabla_{j} u^{j}\right) b^{i}=0
$$


for $\eta=0$, and similarly for equations such as (2.11, 2.13). We have considered the compressible case, as it does allow a clear emergence of the magnetic pressure term. For the incompressible case one drops the internal energy $e(\rho, s)$ and in its place uses a Lagrange multiplier to enforce that the flow map conserves volumes, $\phi_{*} \mu=\mu$. The theory can be developed further from Lagrangian to Hamiltonian mechanics, using a noncanonical Poisson bracket as discussed in Morrison (1982, 1998), Morrison. Andreussi and Pegoraro (2020) and reviewed in Webb (2018), with extensions to relativistic MHD (D'Avignon, Morrison and Pegoraro, 2015) and two-fluid MHD (Lingam. Miloshevich and Morrison, 2016).

\subsection{Helicity}

Ideal fluid mechanics, MHD and extensions involve the Lie transport of vector fields in a flow, and resulting conserved quantities are the helicities that give the linkages between the integral curves or field lines of the various vector fields (see, e.g. Arnold and Khesin, 1998; Fukumoto, 2008; Lingam. Miloshevich and Morrison, 2016; Moffatt and Dormy, 2019, and references therein). Helicities result from Noether's theorem under the symmetry of relabelling Lagrangian coordinates (Padhiye and Morrison, 1996), and Enciso, Peralta-Salas and Torres de Lizal (2016) show that helicity is the only integral invariant of a general vector field transported in volumepreserving flows. Extensions to helicity and linkage in relativistic MHD are given by Yoshida, Kawazura and Yokoyama (2014), and related to the relabelling symmetry in Kawazura. Yoshida and Fukumoto (2014).

In ideal (non-relativistic) MHD we have conservation of magnetic helicity given by the integral of the helicity 3 -form $h_{\mathrm{M}}=\alpha \wedge \mathrm{d} \alpha$ over $\mathcal{M}$ with appropriate boundary conditions. Here $\alpha$ is a 1-form potential for $B$ so that $B=\mathrm{d} \alpha$ and, from (2.8) and in a suitable gauge,

$$
\partial_{t} \alpha+\mathcal{L}_{u} \alpha=0
$$

For this gauge the helicity form is conserved on fluid parcels

$$
\partial_{t}(\alpha \wedge B)+\mathcal{L}_{u}(\alpha \wedge B)=0,
$$

and its integral over $\mathcal{M}$ is independent of gauge with suitable boundary conditions. Explicitly, if we set

$$
H_{\mathrm{M}}=\int_{\mathcal{M}} \alpha \wedge B
$$

then we have

$$
\left.\left.\frac{\mathrm{d} H_{\mathrm{M}}}{\mathrm{d} t}=-\int_{\mathcal{M}} \mathcal{L}_{u}(\alpha \wedge B)=-\int_{\mathcal{M}} \mathrm{d}[u\lrcorner(\alpha \wedge B)\right]=-\int_{\partial \mathcal{M}} u\right\lrcorner(\alpha \wedge B)=0
$$

for $u$ parallel to the boundary $\partial \mathcal{M}$. See Arnold and Khesin (1998), Moffatt and Dormy (2019) for more discussion, for example of gauge invariance. If the magnetic helicity density $h_{\mathrm{M}}=\alpha \wedge B$ vanishes everywhere, the resulting Pfaff integrability condition means that locally $\alpha$ defines a family of surfaces $\Phi=$ const., with $\alpha \propto \mathrm{d} \Phi$, and a further Godbillon-Vey helicity invariant may be defined, as discussed by Webb (2018), Webb et al. (2019) and Machon (2020).

For the cross helicity, the appropriate 3 -form is $h_{\mathrm{C}}=\nu \wedge B$ (in traditional terms $\boldsymbol{u} \cdot \boldsymbol{b}$ ) and is conserved in incompressible, constant density flow. To see this here, scale out $\rho$ and write the momentum and induction equations as

$$
\begin{aligned}
\partial_{t} \nu & +\mathcal{L}_{u} \nu=-\mathrm{d} \pi+\mathcal{L}_{b} \beta, \\
\partial_{t} B & +\mathcal{L}_{u} B=0
\end{aligned}
$$

where $\pi$ absorbs terms such as the magnetic pressure. Then we have

$$
\begin{aligned}
\partial_{t}(\nu \wedge B)+\mathcal{L}_{u}(\nu \wedge B) & =-\mathrm{d} \pi \wedge B+\left(\mathcal{L}_{b} \beta\right) \wedge B \\
& =-\mathrm{d}(\pi B)+\mathcal{L}_{b}(\beta \wedge B) \\
& =-\mathrm{d}(\pi B)+\mathrm{d}[b\lrcorner(\beta \wedge B)],
\end{aligned}
$$


using $\mathrm{d} B=0, \mathcal{L}_{b} B=0\left(\right.$ as $\mathcal{L}_{b} b=0, \mathcal{L}_{b} \mu=0$ and $\left.b\right\lrcorner \mu=B$ ), and Cartan's formula. Again this leads to a conservation law; if we set

$$
H_{\mathrm{C}}=\int_{\mathcal{M}} \nu \wedge B
$$

we have

$$
\left.\left.\left.\frac{\mathrm{d} H_{\mathrm{C}}}{\mathrm{d} t}=-\int_{\partial \mathcal{M}}[-u\lrcorner(\nu \wedge B)-\pi b\right\lrcorner \mu+b\right\lrcorner(\beta \wedge B)\right]=0,
$$

given that $u, b \| \partial \mathcal{M}$.

In MHD the kinetic helicity, the integral of $\boldsymbol{u} \cdot \boldsymbol{\omega}$ in everyday terms, or here the integral of $h_{\mathrm{K}}=\nu \wedge \zeta$ with $\zeta=\mathrm{d} \nu$, is not conserved, but in incompressible flow obeys

$$
\begin{aligned}
\partial_{t}(\nu \wedge \zeta)+\mathcal{L}_{u}(\nu \wedge \zeta) & =-\mathrm{d} \pi \wedge \zeta+\left(\mathcal{L}_{b} \beta\right) \wedge \zeta+\nu \wedge\left(\mathcal{L}_{b} \mathrm{~d} \beta\right) \\
& \simeq 2\left(\mathcal{L}_{b} \beta\right) \wedge \zeta \simeq 2 \nu \wedge\left(\mathcal{L}_{b} \mathrm{~d} \beta\right),
\end{aligned}
$$

using $\simeq$ to discard $\mathrm{d}(\cdot)$ terms. The two versions follow from

$$
\begin{aligned}
\mathrm{d}\left(\mathcal{L}_{b} \beta \wedge \nu\right) & =\mathrm{d}\left(\mathcal{L}_{b} \beta\right) \wedge \nu-\left(\mathcal{L}_{b} \beta\right) \wedge \mathrm{d} \nu \\
& =\left(\mathcal{L}_{b} \mathrm{~d} \beta\right) \wedge \nu-\left(\mathcal{L}_{b} \beta\right) \wedge \mathrm{d} \nu \\
& =\nu \wedge\left(\mathcal{L}_{b} \mathrm{~d} \beta\right)-\left(\mathcal{L}_{b} \beta\right) \wedge \zeta .
\end{aligned}
$$

\section{Pull backs and Alfvén waves}

\subsection{Transformation under a mapping}

In this section we again consider incompressible fluid flow of constant density $\rho$; we rescale the pressure $p$ and magnetic field $B$ so as to absorb this quantity, effectively setting $\rho=1$. We restate the governing equations as

$$
\begin{aligned}
& \partial_{t} \nu+\mathcal{L}_{u} \nu+\mathrm{d} \pi=\mathcal{L}_{b} \beta \\
& \partial_{t} b+\mathcal{L}_{u} b=0
\end{aligned}
$$

with

$$
\begin{aligned}
& \nu=u_{b}, \quad \beta=b_{b}, \\
& \pi=p-\frac{1}{2} g(u, u)+g(b, b), \\
& \operatorname{div} u=0, \quad \operatorname{div} b=0 .
\end{aligned}
$$

In incompressible flow, both field $b$ and flux $B$ are Lie dragged from their initial conditions $b_{0}$ and $B_{0}$ by the flow map $\phi$ which preserves volume:

$$
b=\phi_{*} b_{0}, \quad B=\phi_{*} B_{0}, \quad \phi_{*} \mu=\mu .
$$

We will focus primarily on $b$ below rather than $B$. 2001),

We now decompose the flow map $\phi$ as a composition of volume-preserving maps (Marsden and Shkoller,

$$
\phi=\xi \circ \bar{\phi}
$$

We have in mind a situation where there are waves on a background flow, in which case $\bar{\phi}$ gives the Lagrangian mapping for the background flow $\bar{u}$ with

$$
\bar{u}=\dot{\bar{\phi}} \circ \bar{\phi}^{-1}
$$


and the map $\xi$ is the fluctuating flow map moving particles from the background flow to their final positions, with the velocity field $w$ defined for $\xi$ by

$$
w=\dot{\xi} \circ \xi^{-1} .
$$

We stress that as far as the mathematical development is concerned $\bar{\phi}$ and $\xi$ are simply two mappings, volumepreserving diffeomorphisms of $\mathcal{M}$, neither being required to have any further properties; in particular the map $\xi$ can be of finite amplitude. Note with this that the bar over $\bar{\phi}$ is at present just a label - it is not a mean map here in that we do not need to define any averaging process - we prefer the adjective background for this reason, rather than mean. We are considering a single fluctuating map $\xi$; this will be replaced by a family of maps $\xi^{\iota}$ labelled by $\iota$ in due course, but at present it is a single map. We note that in the literature the decomposition (3.7) is sometimes referred to as the use of hybrid Eulerian-Lagrangian (HEL) coordinates (e.g. Roberts and Soward, 2006a; Soward and Roberts, 2014), in that the background fluid flow $\bar{u}$ (from the map $\bar{\phi}$ ) captures the Eulerian motion, while the family of maps $\xi^{\iota}$ captures subsequent Lagrangian displacements, which we refer to as fluctuations for brevity.

We can apply the pull back $\xi^{*}$ to carry fluid parcels from their positions given by $\phi$ at time $t$, to their positions in the background flow, given by $\bar{\phi}$. This carries magnetic quantities according to the Cauchy solution and likewise for forms such as $B$ and $\nu$. For magnetic field we can define

$$
\bar{b}=\xi^{*} b=\bar{\phi}_{*} b_{0}, \quad \bar{B}=\xi^{*} B=\bar{\phi}_{*} B_{0},
$$

where the second equalities, stemming from (3.6), indicate that the pulled back fields $\bar{b}$ and $\bar{B}$ are just the push forwards of the initial condition by the background flow map $\bar{\phi}$ and so are independent of $\xi$. For the flow, differentiating (3.7) shows that

$$
u=\xi_{*} \bar{u}+w, \quad \bar{u}=\xi^{*}(u-w) .
$$

Thus the background flow $\bar{u}$, pushed forward by $\xi$, and the fluctuation velocity $w$ sum to give the flow velocity at any point.

For other quantities we set

$$
\tilde{\nu}=\xi^{*} \nu, \quad \tilde{\pi}=\xi^{*} \pi, \quad \tilde{\beta}=\xi^{*} \beta, \quad \tilde{g}=\xi^{*} g, \quad \tilde{\mu}=\xi^{*} \mu,
$$

so that the tilde is a label for these and other quantities obtained by applying $\xi^{*}$. Note that the background fields $\bar{u}$ and $\bar{b}$ depend only on the background flow map $\bar{\phi}$ (and for $\bar{b}$ the initial condition), but this is not the case for $\tilde{\nu}, \tilde{\pi}, \tilde{\beta}$, hence the distinct notation. This becomes relevant below when we have an ensemble of maps, say $\xi^{\iota}$.

Applying $\xi^{*}$ to the equations for $\nu$ and $b$ and using standard identities (Gilbert and Vanneste, 2018) gives

$$
\begin{aligned}
& \partial_{t} \tilde{\nu}+\mathcal{L}_{\bar{u}} \tilde{\nu}+\mathrm{d} \tilde{\pi}=\mathcal{L}_{\bar{b}} \tilde{\beta}, \\
& \partial_{t} \bar{b}+\mathcal{L}_{\bar{u}} \bar{b}=0 .
\end{aligned}
$$

The induction equation just simplifies to (3.14), namely motion of the background field $\bar{b}$ in the background flow $\bar{u}$, in other words all trace of the fluctuations has vanished. However for the momentum equation, originally in (3.1) we had transport of $\nu=u_{b}$ in the flow $u$ : momentum $\nu$ and velocity $u$ were simply related by the metric $g$ at each point. Now, in (3.13) the momentum and flow are not so easily related. We have instead that

$$
\tilde{\nu}=\xi^{*} \nu=\xi^{*}[g(u, \cdot)]=\xi^{*}\left[g\left(\xi_{*} \bar{u}+w, \cdot\right)\right]=\tilde{g}(\bar{u}, \cdot)+\tilde{g}(\tilde{w}, \cdot),
$$

and for $\beta$,

$$
\tilde{\beta}=\xi^{*} \beta=\xi^{*}[g(b, \cdot)]=\tilde{g}(\bar{b}, \cdot),
$$

and so the relation involves the background flow for $\tilde{\nu}$ and the pulled-back metric $\tilde{g}=\xi^{*} g$ for both. Thus, the momentum equation involves transport, not of the momentum of the background flow which would be $(\bar{u})_{b}$ but transport of a quantity $\tilde{\nu}$ that differs from this, and likewise for $(\bar{b})_{b}$ and $\tilde{\beta}$. For the magnetic field, the difference $\tilde{g}(\bar{b}, \cdot)-(\bar{b})_{b}$ between the two expressions captures the difference between turning the vector field $\bar{b}$ into a 1-form field using the metric $g$ or the pulled back metric $\tilde{g}$. For the momentum $\tilde{\nu}$, there is an additional term arising from the fluctuating flow itself. 


\subsection{Lagrangian averaging}

In this section we will make some comments about how the above framework is used for Lagrangian averaging, with an emphasis on MHD; see Holm (2002a) and Gilbert and Vanneste (2018) for further discussion. We suppose that we have an ensemble of fluctuating maps $\xi^{\iota}$, labelled by $\iota$, on the same background flow given by $\bar{\phi}$ or $\bar{u}$. We also assume that we have the same initial magnetic field in each realisation of the ensemble, so that barred quantities such as $\bar{u}, \bar{B}$ and $\bar{b}$ are independent of $\iota$. On the other hand, tilded quantities do depend on $\iota$ and we can label these with an $\iota$ to stress this if we need to (we do this sparingly). For any tensor quantity, say $\tau^{\iota}$. we can define its Lagrangian average as

$$
\bar{\tau}^{\mathrm{L}}=\left\langle\xi^{\iota *} \tau^{\iota}\right\rangle,
$$

where $\langle\cdot\rangle$ denotes an ensemble average. For barred quantities, we have

$$
\bar{b}^{\mathrm{L}}=\bar{b} \quad \text { and } \quad \bar{B}^{\mathrm{L}}=\bar{B},
$$

by virtue of (3.10). In contrast,

$$
\bar{u}^{\mathrm{L}} \neq \bar{u}
$$

in general, since $\bar{u}$ is defined in terms of the map $\bar{\phi}$ by (3.8) and not in terms of a Lagrangian average; as argued by Gilbert and Vanneste (2018), the equality cannot hold for any useful definition of the mean flow map $\bar{\phi}$. Note that in (3.17) any tensor quantity is transported from the locations $\phi^{\iota}(x)$ of a single Lagrangian parcel in each realisation to a single point $\bar{\phi}(x)$ in $\mathcal{M}$ and then averaged. Thus averaging always takes place in the tangent space, co-tangent space and tensor spaces at each point. We never attempt to average vectors and tensors located at different points of $\mathcal{M}$, as this cannot be defined in general. (The generalised Lagrangian mean theory of Andrews and McIntyre (1978a,b) (see also Bühler (2009)) assumes a Euclidean structure to average vectors based at different points using parallel translation.)

Applying this Lagrangian average to the incompressible MHD equations (3.13, 3.14), and letting barred quantities come out of any averaging, we have

$$
\begin{aligned}
\partial_{t} \bar{\nu}^{\mathrm{L}}+\mathcal{L}_{\bar{u}} \bar{\nu}^{\mathrm{L}}+\mathrm{d} \bar{\pi}^{\mathrm{L}}=\mathcal{L}_{\bar{b}} \bar{\beta}^{\mathrm{L}}, \\
\partial_{t} \bar{b}+\mathcal{L}_{\bar{u}} \bar{b}=0 .
\end{aligned}
$$

The Lagrangian averaged momentum $\bar{\nu}^{\mathrm{L}}$ and $\bar{\beta}^{\mathrm{L}}$ differ from the similar background quantities by what are known as the pseudomomentum $\mathrm{p}$ and the pseudofield $\mathrm{h}$ respectively; these are given by

$$
-\mathrm{p}=\bar{\nu}^{\mathrm{L}}-(\bar{u})_{\mathrm{b}}, \quad-\mathrm{h}=\bar{\beta}^{\mathrm{L}}-(\bar{b})_{b} .
$$

(the minus sign for $\mathrm{p}$ is a convention; see Andrews and McIntyre (1978a)). In the context of GLM theory, Holm (2002a) also identifies $h$ and refers to its average, over an ensemble of flows, as the magnetization induced by Lagrangian averaging. The pseudomomentum and pseudofield capture the effect of the fluctuations on the background flow; explicit expressions in terms of $\xi^{\iota}$ can be obtained by averaging (3.15, 3.16). We give an example in the next section.

We finally consider the effect of a pull back and Lagrangian averaging on the various helicities discussed in section 2.5. We consider first cross helicity (Holm, 2002a), applying a pull back to equation (2.58) to obtain

$$
\left.\partial_{t}(\tilde{\nu} \wedge \bar{B})+\mathcal{L}_{\bar{u}}(\tilde{\nu} \wedge \bar{B})=-\mathrm{d}(\tilde{\pi} \bar{B})+\mathrm{d}[\bar{b}\lrcorner(\tilde{\beta} \wedge \bar{B})\right]
$$

taking an average gives

$$
\left.\partial_{t}\left(\bar{\nu}^{\mathrm{L}} \wedge \bar{B}\right)+\mathcal{L}_{\bar{u}}\left(\bar{\nu}^{\mathrm{L}} \wedge \bar{B}\right)=-\mathrm{d}\left(\bar{\pi}^{\mathrm{L}} \bar{B}\right)+\mathrm{d}[\bar{b}\lrcorner\left(\bar{\beta}^{\mathrm{L}} \wedge \bar{B}\right)\right] .
$$

Thus we can define the Lagrangian averaged cross helicity form by ${\overline{h_{\mathrm{C}}}}^{\mathrm{L}}=\bar{\nu}^{\mathrm{L}} \wedge \bar{B}$ and note that it differs from that of the background fields, namely $(\bar{u})_{b} \wedge \bar{B}$ by a term involving the pseudomomentum, namely $-\mathrm{p} \wedge \bar{B}$. 
The cross helicity form obeys the above transport equation. This means that for any subvolume $\mathcal{V}$ of $\mathcal{M}$ we can account for the change of cross helicity in $\mathcal{V}$ by fluxes across the boundary $\mathcal{S}=\partial \mathcal{V}$

$$
\left.\left.\partial_{t} \int_{\mathcal{V}} \bar{\nu}^{\mathrm{L}} \wedge \bar{B}=\int_{\mathcal{S}}[-\bar{u}\lrcorner\left(\bar{\nu}^{\mathrm{L}} \wedge \bar{B}\right)-\bar{\pi}^{\mathrm{L}} \bar{B}+\bar{b}\right\lrcorner\left(\bar{\beta}^{\mathrm{L}} \wedge \bar{B}\right)\right] .
$$

Applying a Lagrangian average to the magnetic helicity simply gives ${\overline{h_{\mathrm{M}}}}^{\mathrm{L}}=\bar{\alpha} \wedge \mathrm{d} \bar{\alpha}$, since the potential $\alpha$ is transported by the flow: $\xi^{*} \alpha=\bar{\alpha}=\bar{\phi}_{*} \alpha_{0}$, so nothing is gained by Lagrangian averaging: no linkages are changed in ideal evolution. Applying a Lagrangian average to the kinetic helicity gives $\bar{h}_{\mathrm{K}}^{\mathrm{L}}=\overline{\nu \wedge}^{\mathrm{L}}$, which does not seem open to any useful simplifications in MHD, as $\zeta=d \nu$ is no longer given by the Cauchy solution. In pure hydrodynamics $(B=0)$, if all realisations in the ensemble have the same initial vorticity field (maybe only locally, if not globally) we can write $\xi^{*} \zeta=\bar{\zeta}=\bar{\phi}_{*} \zeta_{0}$ and so $\bar{h}_{\mathrm{K}}^{\mathrm{L}}=\bar{\nu}^{\mathrm{L}} \wedge \bar{\zeta}$. Again this differs from the kinetic helicity of the background fields, namely $(\bar{u})_{b} \wedge \bar{\zeta}$, by a term involving the pseudomomentum, $-\mathrm{p} \wedge \bar{\zeta}$, Helicity conservation is expressed by

$$
\left.\partial_{t} \int_{\mathcal{V}} \bar{\nu}^{\mathrm{L}} \wedge \bar{\zeta}=\int_{\mathcal{S}}[-\bar{u}\lrcorner\left(\bar{\nu}^{\mathrm{L}} \wedge \bar{\zeta}\right)-\bar{\pi}^{\mathrm{L}} \bar{\zeta}\right]
$$

which is zero if the background vorticity $\bar{\zeta}$ vanishes on $\mathcal{S}$. Thus, for cross helicity in MHD and for kinetic helicity in hydrodynamics, the helicity 'hidden' in the fluctuations is easily expressed in terms of the pseudomomentum.

\subsection{Alfvén waves}

As a fundamental example we consider how a pull back can be employed for an Alfvén wave on a uniform magnetic field in Euclidean geometry, $\mathcal{M}=\mathbb{R}^{3}$ with coordinates $(x, y, z)=\left(x^{1}, x^{2}, x^{3}\right)$ and the usual metric $g=\mathrm{d} x^{2}+\mathrm{d} y^{2}+\mathrm{d} z^{2}$. In ideal MHD with a uniform background magnetic field, waves of arbitrary shape can propagate without change, with the velocity and disturbance magnetic field proportional to each other, as found by Walén (1944) (see, e.g. Alfvén and Falthammar, 1950). We consider a flow and magnetic field giving such a travelling wave by setting

$$
f=f(z-c t), \quad h=h(z-c t),
$$

and then

$$
\begin{aligned}
& u=f \partial_{x}+h \partial_{y}+\mathscr{U} \partial_{z}, \quad b= \pm f \partial_{x} \pm h \partial_{y}+\mathscr{B} \partial_{z}, \\
& \nu=f \mathrm{~d} x+h \mathrm{~d} y+\mathscr{U} \mathrm{d} z, \quad \beta= \pm f \mathrm{~d} x \pm h \mathrm{~d} y+\mathscr{B} \mathrm{d} z
\end{aligned}
$$

where $\mathscr{U}$ and $\mathscr{B}$ are constant uniform flow and field respectively, $f$ and $h$ are arbitrary functions, and the wave speed

$$
c=\mathscr{U} \mp \mathscr{B} .
$$

It is easy to check that this satisfies (3.1, 3.2 with $\pi=0$ by manipulating the 1-forms $\nu$ and $\beta$ directly, using the commutation of Lie and exterior derivatives:

$$
\begin{aligned}
\partial_{t} \nu+\mathcal{L}_{u} \nu & =-c\left(f^{\prime} \mathrm{d} x+h^{\prime} \mathrm{d} y\right)+\mathcal{L}_{u} f \mathrm{~d} x+\mathcal{L}_{u} h \mathrm{~d} y+\mathcal{L}_{u} \mathscr{U} \mathrm{d} z+f \mathrm{~d}\left(\mathcal{L}_{u} x\right)+h \mathrm{~d}\left(\mathcal{L}_{u} y\right)+\mathscr{U} \mathrm{d}\left(\mathcal{L}_{u} z\right) \\
& =(\mathscr{U}-c) f^{\prime} \mathrm{d} x+(\mathscr{U}-c) h^{\prime} \mathrm{d} y+f \mathrm{~d} f+h \mathrm{~d} h \\
\mathcal{L}_{b} \beta & = \pm \mathcal{L}_{b} f \mathrm{~d} x \pm \mathcal{L}_{b} h \mathrm{~d} y+\mathcal{L}_{b} \mathscr{B} \mathrm{d} z \pm f \mathrm{~d}\left(\mathcal{L}_{b} x\right) \pm h \mathrm{~d}\left(\mathcal{L}_{b} y\right)+\mathscr{B} \mathrm{d}\left(\mathcal{L}_{b} z\right) \\
& = \pm \mathscr{B} f^{\prime} \mathrm{d} x \pm \mathscr{B} h^{\prime} \mathrm{d} y+f \mathrm{~d} f+h \mathrm{~d} h
\end{aligned}
$$

We now map the vector field $u$ in (3.28) onto a background flow chosen as $\bar{u}=\mathscr{U} \partial_{z}$; in other words, we remove the Alfvén wave by the pull back. This is achieved with fluctuations of the form

$$
\xi(x, y, z, t)=(x+F(z-c t), y+H(z-c t), z) .
$$


and associated velocity

$$
w=-c F^{\prime} \partial_{x}-c H^{\prime} \partial_{y} .
$$

We can use (3.11), that is, $\bar{u}=\xi^{*}(u-w)$, to compute $\bar{u}$. Noting that

$$
\tilde{\partial_{x}}=\xi^{*} \partial_{x}=\partial_{x}, \quad \tilde{\partial_{y}}=\xi^{*} \partial_{y}=\partial_{y}, \quad \tilde{\partial_{z}}=\xi^{*} \partial_{z}=-F^{\prime} \partial_{x}-H^{\prime} \partial_{y}+\partial_{z},
$$

we find

$$
\bar{u}=\left(f \mp \mathscr{B} F^{\prime}\right) \partial_{x}+\left(h \mp \mathscr{B} H^{\prime}\right) \partial_{y}+\mathscr{U} \partial_{z},
$$

hence we choose

$$
F^{\prime}= \pm \mathscr{B}^{-1} f, \quad H^{\prime}= \pm \mathscr{B}^{-1} h .
$$

With this choice, the background magnetic field, obtained from (3.10) as

$$
\bar{b}=\left( \pm f-\mathscr{B} F^{\prime}\right) \partial_{x}+\left( \pm h-\mathscr{B} H^{\prime}\right) \partial_{y}+\mathscr{B} \partial_{z},
$$

also simplifies, leading to the uniform background vector fields

$$
\bar{u}=\mathscr{U} \partial_{z}, \quad \bar{b}=\mathscr{B} \partial_{z},
$$

which trivially satisfy the induction equation (3.14). The corresponding 1-forms, in contrast, are more complicated. Using that

$$
\tilde{\mathrm{d} x}=\xi^{*} \mathrm{~d} x=\mathrm{d}\left(\xi^{*} x\right)=\mathrm{d} x+F^{\prime} \mathrm{d} z, \quad \tilde{\mathrm{d}} y=\xi^{*} \mathrm{~d} y=\mathrm{d}\left(\xi^{*} y\right)=\mathrm{d} y+H^{\prime} \mathrm{d} z, \quad \tilde{\mathrm{d}} z=\xi^{*} \mathrm{~d} z=\mathrm{d}\left(\xi^{*} z\right)=\mathrm{d} z,
$$

we obtain

$$
\begin{aligned}
& \tilde{\nu}=f \mathrm{~d} x+h \mathrm{~d} y+\left[\mathscr{U} \pm \mathscr{B}^{-1}\left(f^{2}+h^{2}\right)\right] \mathrm{d} z, \\
& \tilde{\beta}= \pm f \mathrm{~d} x \pm h \mathrm{~d} y+\left[\mathscr{B}+\mathscr{B}^{-1}\left(f^{2}+h^{2}\right)\right] \mathrm{d} z,
\end{aligned}
$$

which depend explicitly on the waves through $f$ and $h$. It can be checked that (3.41, 3.42) solve the pulledback momentum equation (3.13).

If we have a sea of Alfvén waves with random phases such that $\langle f\rangle=\langle h\rangle=0$, then we can take a Lagrangian average as outlined above to replace previously tilded quantities, and obtain

$$
\begin{aligned}
\bar{\nu}^{\mathrm{L}} & =\left[\mathscr{U} \pm \mathscr{B}^{-1}\left\langle f^{2}+h^{2}\right\rangle\right] \mathrm{d} z, \quad \bar{\beta}^{\mathrm{L}}=\left[\mathscr{B}+\mathscr{B}^{-1}\left\langle f^{2}+h^{2}\right\rangle\right] \mathrm{d} z \\
-\mathrm{p} & = \pm \mathscr{B}^{-1}\left\langle f^{2}+h^{2}\right\rangle \mathrm{d} z, \quad-\mathrm{h}=\mathscr{B}^{-1}\left\langle f^{2}+h^{2}\right\rangle \mathrm{d} z .
\end{aligned}
$$

Thus we gain a correction to the $z$-directed momentum of the background flow $(\bar{u})_{b}=\mathscr{U} \mathrm{d} z$ and to the mean 1-form field $(\bar{b})_{b}=\mathscr{B} \mathrm{d} z$ that parameterises these waves, and we identify the pseudomomentum and pseudofield as quantities that are quadratic in the wave amplitude, and have only a $z$ component. The cross helicity carried by the waves (with the background contribution subtracted out) is

$$
-\mathrm{p} \wedge \bar{B}= \pm\left\langle f^{2}+h^{2}\right\rangle \mathrm{d} x \wedge \mathrm{d} y \wedge \mathrm{d} z
$$

The above example is straightforward since the Alfvén waves are wholly transverse and the nonlinear terms cancel out; the results are equivalent to what would be obtained by GLM theory and averaging over the phases of the waves. Nonetheless this example does capture how a mapping can straighten out magnetic field lines and encode the disturbance in the pseudo-quantities. Extension to non-uniform magnetic fields and compressible flows, in order to understand wave-mean flow/field interactions (see Bühler, 2009), are the subject of future study, beyond the scope of the present paper. 


\section{The Braginsky dynamo}

As a second example of the use of a geometric approach to MHD, we consider the Braginsky dynamo. We will allow for compressible flow $u$ in this section. As a kinematic dynamo, the flow field $u$ is specified and for non-zero, uniform magnetic diffusion $\eta>0$, the induction equation (2.8) becomes

$$
\partial_{t} B+\mathcal{L}_{u} B=\eta \nabla^{2} B
$$

Here, the operator $\nabla^{2}$ is (minus) the Laplace-de Rham operator,

$$
-\nabla^{2}=\delta \mathrm{d}+\mathrm{d} \delta
$$

where $\delta \equiv \star \mathrm{d} \star$ is the codifferential operator, here mapping the 2-form $B$ to a 1-form. We have already identified $j=\mathrm{d} \beta=\mathrm{d} \star B$ as the current 2-form, and thus the operator $\mathrm{d} \star$ corresponds to a curl. Since $\mathrm{d} B=0$,

$$
\nabla^{2} B=-\mathrm{d} \delta B=-\mathrm{d} \star \mathrm{d} \star B
$$

is equivalent to the two curls in (2.2, 2.3).

In kinematic dynamo theory, the aim is to show that growing solutions exist for a particular choice of flow and diffusivity $\eta>0$. Our goal is to obtain formulae for the transport term known as the $\alpha$-effect, by exploiting the machinery of pull-backs and operators in a general setting, rather than the explicit construction of $\alpha \omega$-dynamos, or explicit calculation of $\alpha$ for particular families of fluctuations or waves. We stress that the overarching ideas behind the $\alpha$-effect and $\alpha \omega$-dynamos are well established and it is only our approach that is novel; for comprehensive discussion, as well as solutions in particular examples, we refer to the book Moffatt and Dormy (2019).

\subsection{Informal approach: an $\alpha \omega$ dynamo}

We first illustrate the ideas underlying the Braginsky dynamo and their geometric formulation by considering a specific scenario. We use coordinates $\left(x^{1}, x^{2}, x^{3}\right)=(x, y, z)$; these need not be Cartesian, nor need $(x, y)$ even be orthogonal, but we do assume that the metric is independent of $z$ taking the form

$$
g(x, y)=\left(\begin{array}{ccc}
g_{11} & g_{12} & 0 \\
g_{21} & g_{22} & 0 \\
0 & 0 & g_{33}
\end{array}\right) .
$$

Thus $g$ is invariant under translations in the $z$-direction and under the transformation $z \rightarrow-z$; examples include spherical polar coordinates $(r, \theta, \phi)$ with $z \equiv \phi$ the azimuthal angle or longitude. The volume form $\mu=|g|^{1 / 2} \mathrm{~d} x \wedge \mathrm{d} y \wedge \mathrm{d} z$ is also $z$-independent. For convenience we set $\varsigma(x, y)=|g|^{-1 / 2}$, with $|g|$ the determinant of $g$, so that the induced volume form is

$$
\mu=\varsigma^{-1} \mathrm{~d} x \wedge \mathrm{d} y \wedge \mathrm{d} z
$$

We let the magnetic flux 2 -form $B$ be written as

$$
B=B_{1} \mathrm{~d} y \wedge \mathrm{d} z+B_{2} \mathrm{~d} z \wedge \mathrm{d} x+B_{3} \mathrm{~d} x \wedge d y .
$$

Note that when we use index notation below we will also use $B_{i j}$ with $B_{1}=B_{23}=-B_{32}$ etc., that is $B=\frac{1}{2} B_{i j} \mathrm{~d} x^{i} \wedge \mathrm{d} x^{j}$, there being little risk of confusion. The condition that $B$ is solenoidal, $\mathrm{d} B=0$, amounts simply to

$$
\partial_{x} B_{1}+\partial_{y} B_{2}+\partial_{z} B_{3}=0
$$

valid for any coordinates. We need the Hodge star operator $\star$, which in three dimensions gives an isomorphism between 1-forms and 2-forms involving the metric and volume form. Starting with the basis 2-forms, we readily find

$$
\mathrm{d} y \wedge \mathrm{d} z \stackrel{\star}{\longleftrightarrow} g_{11} \varsigma \mathrm{d} x+g_{21} \varsigma \mathrm{d} y, \quad \mathrm{~d} z \wedge \mathrm{d} x \stackrel{\star}{\longleftrightarrow} g_{12} \varsigma \mathrm{d} x+g_{22} \varsigma \mathrm{d} y, \quad \mathrm{~d} x \wedge \mathrm{d} y \stackrel{\star}{\longleftrightarrow} g_{33} \varsigma \mathrm{d} z .
$$


We are now ready to discuss the operation of an $\alpha \omega$-dynamo, which we build step by step.

First consider a flow in the $z$-direction only, of the form

$$
u=u^{3}(x, y) \partial_{z} .
$$

The induction equation (4.1) with $\eta=0$ becomes

$$
\begin{aligned}
0 & =\left(\partial_{t}+\mathcal{L}_{u}\right)\left(B_{1} \mathrm{~d} y \wedge \mathrm{d} z+B_{2} \mathrm{~d} z \wedge \mathrm{d} x+B_{3} \mathrm{~d} x \wedge \mathrm{d} y\right) \\
& =\left[\left(\partial_{t}+u^{3} \partial_{z}\right) B_{1}\right] \mathrm{d} y \wedge \mathrm{d} z+B_{1} \mathrm{~d} y \wedge \mathrm{d} u^{3}+\left[\left(\partial_{t}+u^{3} \partial_{z}\right) B_{2}\right] \mathrm{d} z \wedge \mathrm{d} x+B_{2} \mathrm{~d} u^{3} \wedge d x \\
& +\left[\left(\partial_{t}+u^{3} \partial_{z}\right) B_{3}\right] \mathrm{d} x \wedge \mathrm{d} y,
\end{aligned}
$$

hence, in components,

$$
\left(\partial_{t}+u^{3} \partial_{z}\right) B_{1}=0, \quad\left(\partial_{t}+u^{3} \partial_{z}\right) B_{2}=0, \quad\left(\partial_{t}+u^{3} \partial_{z}\right) B_{3}=\left(B_{1} \partial_{x}+B_{2} \partial_{y}\right) u^{3} .
$$

On the left-hand side we have transport of field components in the flow $u$, and on the right-hand side of the final equation we observe the well-known $\omega$-effect, namely the generation of the azimuthal $B_{3}$ component from transverse components $B_{1}$ and $B_{2}$. Given the simplicity of the flow field, there is no term that regenerates $B_{1}$ and $B_{2}$ field.

We now include diffusion $\eta>0$ and see what diffusive processes can convert $B_{3}(x, y)$ field into $B_{1}, B_{2}$ components. Making use of (4.8), we calculate the right-hand side of (3.21) applied only to this component as follows:

$$
\begin{aligned}
B & =B_{3}(x, y) \mathrm{d} x \wedge \mathrm{d} y \\
\star B & =g_{33} \varsigma B_{3} \mathrm{~d} z \\
\mathrm{~d} \star B & =\left(g_{33} \varsigma B_{3}\right)_{y} \mathrm{~d} y \wedge \mathrm{d} z-\left(g_{33} \varsigma B_{3}\right)_{x} \mathrm{~d} z \wedge \mathrm{d} x \\
\delta B=\star \mathrm{d} \star B & =\left[g_{11} \varsigma\left(g_{33} \varsigma B_{3}\right)_{y}-g_{12} \varsigma\left(g_{33} \varsigma B_{3}\right)_{x}\right] \mathrm{d} x+\left[g_{21} \varsigma\left(g_{33} \varsigma B_{3}\right)_{y}-g_{22} \varsigma\left(g_{33} \varsigma B_{3}\right)_{x}\right] \mathrm{d} y, \\
\mathrm{~d} \delta B=\mathrm{d} \star \mathrm{d} \star B & =\left[\left[g_{21} \varsigma\left(g_{33} \varsigma B_{3}\right)_{y}-g_{22} \varsigma\left(g_{33} \varsigma B_{3}\right)_{x}\right]_{x}-\left[g_{11} \varsigma\left(g_{33} \varsigma B_{3}\right)_{y}-g_{12} \varsigma\left(g_{33} \varsigma B_{3}\right)_{x}\right]_{y}\right] \mathrm{d} x \wedge \mathrm{d} y .
\end{aligned}
$$

Thus, the diffusion of $B_{3}(x, y)$ field in this geometry does not lead to $B_{1}, B_{2}$ components as defined in (4.6).

If the $B_{3}$ field depends additionally on $z$ then there is the generation of $B_{1}, B_{2}$ components, but a field depending on $z$ is liable to enhanced diffusion because of the effect of $u^{3}(x, y)$ in (4.9) in reducing transverse scales and enhancing dissipation. Instead, aiming for an $\alpha \omega$-dynamo in the traditional formulation, we specify a field $B_{3}(x, y)$ that is independent of $z$ and so robust to this process, and seek other mechanisms. In the Braginsky dynamo, the idea is to replace the flow in (4.9) by something more complicated, namely by adding some finite amplitude $z$-dependent motion to the flow field.

Within the context of our geometric approach, we consider now the flow map $\phi$ for the velocity field $u$ to be written as

$$
\phi=\xi \circ \bar{\phi}
$$

where the background flow $\bar{u}=\dot{\bar{\phi}} \circ \bar{\phi}^{-1}$ again takes the simple form

$$
\bar{u}=\bar{u}_{3}(x, y) \partial_{z}
$$

and $\xi$ is a general, time-dependent, finite amplitude map; in particular $\xi$ can have arbitrary $z$-dependence. We can have in mind for example that the full flow $u$ consists of finite-amplitude waves on a simple background shear flow $\bar{u}$. Applying $\xi^{*}$ to the induction equation (4.1) gives us the pulled back version

$$
\partial_{t} \tilde{B}+\mathcal{L}_{\bar{u}} \tilde{B}=-\eta \xi^{*}(\mathrm{~d} \delta B) .
$$

We use $\tilde{B}=\xi^{*} B$ rather than $\bar{B}$ in an earlier section, since with diffusion $\eta>0$ we no longer have the Cauchy solution to relate the magnetic field to its initial condition. Equations (4.11) are now relevant for the effect of the flow $\bar{u}(4.18)$ ) on the field $\tilde{B}$ in the absence of dissipation, $\eta=0$, and we have the $\omega$-effect acting 
on the $\tilde{B}_{1}$ and $\tilde{B}_{2}$ components to generate a $\tilde{B}_{3}$ component; the effect of the distortions to the flow via $\xi$ has completely vanished from the left hand side. The effect though is present on the right-hand side as the Hodge star operator involves the volume form and metric (as in (4.8)), and under the pull back we can write

$$
\xi^{*}(\mathrm{~d} \delta B)=\xi^{*}(\mathrm{~d} \star \mathrm{d} \star B)=\mathrm{d} \tilde{\star} \mathrm{d} \tilde{\star} \tilde{B}
$$

where $\tilde{\star}$ applies the star using the pulled back metric $\tilde{g}$ and volume form $\tilde{\mu}$. We recall how the Hodge star operator works, here in this pulled-back version. We take a 2 -form field $\tilde{B}$ and generate the corresponding vector field $\tilde{b}$ via $\tilde{b}\lrcorner \tilde{\mu}=\tilde{B}$. We then use the flat operator to give us a 1 -form field $\tilde{\beta}$ via $\tilde{\beta}=\tilde{g}(\tilde{b}, \cdot)$. This field is $\tilde{\beta}=\tilde{\star} \tilde{B}$ and provided the pulled back metric $\tilde{g}$ is sufficiently complicated, i.e. the fluctuations coded in $\xi$ break enough symmetry, then we would expect (4.20) in (4.19) to generate $\tilde{B}_{1}$ and $\tilde{B}_{2}$ components from $\tilde{B}_{3}(x, y)$ - the origin of the $\alpha$-effect.

To make further progress we write these Hodge star calculations in coordinates as:

$$
\tilde{b}^{i} \tilde{\varsigma}^{-1} \varepsilon_{i j k}=\tilde{B}_{j k}, \quad \tilde{b}^{i}=\tilde{\varsigma} \frac{1}{2} \varepsilon^{i j k} \tilde{B}_{j k}, \quad \tilde{\beta}_{i}=\tilde{g}_{i j} \tilde{b}^{j}, \quad \tilde{\beta}_{i}=\tilde{g}_{i j} \tilde{\varsigma} \frac{1}{2} \varepsilon^{j k l} \tilde{B}_{k l},
$$

where $\varepsilon_{i j k}$ or $\varepsilon^{i j k}$ is the usual Levi-Civita alternating symbol, and we recall the discussion below (4.6) about components written as $\tilde{B}_{i}$ versus $\tilde{B}_{j k}$. Also we have for the action of d on any 1 -form $\lambda$,

$$
(\mathrm{d} \lambda)_{i j}=2 \partial_{[i} \lambda_{j]}=\partial_{i} \lambda-\partial_{j} \lambda,
$$

where $[\cdot]$ is antisymmetrisation. With this we can write (for any magnetic flux 2-form $\tilde{B}$ ) the components of the (pulled back) electromotive force1 (emf) $\tilde{\mathcal{E}}$, defined by

$$
\tilde{\mathcal{E}}=-\eta \xi^{*}(\delta B)=-\eta \tilde{\star} \mathrm{d} \tilde{\star} \tilde{B},
$$

as

$$
\begin{aligned}
\tilde{\mathcal{E}}_{i} & =-\eta \tilde{g}_{i j} \tilde{\varsigma} \varepsilon^{j k l} \partial_{[k}\left(\tilde{g}_{l] m} \tilde{\varsigma} \frac{1}{2} \varepsilon^{m n p} \tilde{B}_{n p}\right) \\
& =-\eta \tilde{g}_{i j} \tilde{\varsigma} \varepsilon^{j k l} \partial_{k}\left(\tilde{g}_{l m} \tilde{\varsigma}\right) \frac{1}{2} \varepsilon^{m n p} \tilde{B}_{n p}-\eta \tilde{g}_{i j} \tilde{\varsigma} \varepsilon^{j k l} \tilde{\varsigma} \tilde{g}_{l m} \frac{1}{2} \varepsilon^{m n p} \partial_{k} \tilde{B}_{n p}
\end{aligned}
$$

(in the last line the antisymmetrisation is dropped, being redundant because of the $\varepsilon^{j k l}$ term). So far this is exact, providing the diffusion operator for any distortion $\xi$ of the coordinate system taking the background flow $\bar{u}$ and field $\tilde{B}$ to the actual, wavey fields, $u$ and $B$. All the complexity is of course hidden in the pull back $\xi^{*}$, giving the tilde fields, as it involves the coordinate map and its derivatives.

We now consider as input a field $\tilde{B}=\tilde{B}_{3}(x, y) \mathrm{d} z$, with $\tilde{B}_{12}=-\tilde{B}_{21}=\tilde{B}_{3}$, etc. This corresponds to taking $m=3$ in the above equation (and $n, p$ are 1 or 2). We are also interested in sources of field for the transverse $\tilde{B}_{1}$ and $\tilde{B}_{2}$ components, which corresponds to taking $i=3$ in the above, as we shall see. Thus, our focus is on:

$$
\begin{aligned}
\tilde{\mathcal{E}}_{3}=-\eta(\tilde{\star} d \tilde{\star} \tilde{B})_{3} & =-\eta \tilde{g}_{3 j} \tilde{\varsigma} \varepsilon^{j k l} \partial_{k}\left(\tilde{g}_{l 3} \tilde{\varsigma}\right) \tilde{B}_{3}-\eta \tilde{g}_{3 j} \tilde{\varsigma} \varepsilon^{j k l} \tilde{\varsigma} \tilde{g}_{l 3} \partial_{k} \tilde{B}_{3} \\
& =-\eta \tilde{g}_{3 j} \tilde{\varsigma} \varepsilon^{j k l} \partial_{k}\left(\tilde{g}_{l 3} \tilde{\varsigma}\right) \tilde{B}_{3} .
\end{aligned}
$$

The second term on the right hand side of (4.26) vanishes by symmetry to leave (4.27); the remaining term in (4.27) vanishes if the metric $\tilde{g}$ takes the unperturbed form (4.4), as indeed it must, but in the presence of some non-trivial distortion of the coordinate system $\xi$ will generally be non-zero. Suppose finally that we have a family of such waves, given by $\xi^{\iota}$, that are translation invariant in $z$ (while $\tilde{B}_{3}$ is independent of $z$ as above). Then if we average the term over such waves we obtain a quantity in (4.26, 4.27) that is $z$-independent and takes the form

$$
\tilde{\mathcal{E}}_{3}=-\eta\left\langle(\tilde{\star} \mathrm{d} \tilde{\star} \tilde{B})_{3}\right\rangle=\alpha \tilde{B}_{3},
$$

\footnotetext{
${ }^{1}$ This should perhaps be referred to as an effective emf as it emerges from the current as a component of the magnetic diffusion term, with $\mathcal{E}=-\eta \star j, \tilde{\mathcal{E}}=-\eta \tilde{\star} \tilde{j}$, and so includes all the usual diffusive effects, rather than being defined from taking the average of $\boldsymbol{u} \times \boldsymbol{b}$ in the traditional formulation; see Soward and Roberts (2014).
} 
with

$$
\alpha(x, y)=-\eta\left\langle\tilde{g}_{3 j} \tilde{\varsigma} \varepsilon^{j k l} \partial_{k}\left(\tilde{g}_{l 3} \tilde{\varsigma}\right)\right\rangle .
$$

Since

$$
\mathrm{d}\left(\tilde{\mathcal{E}}_{3} \mathrm{~d} z\right)=\mathrm{d}\left(\alpha \tilde{B}_{3} \mathrm{~d} z\right)=\left(\alpha \tilde{B}_{3}\right)_{y} \mathrm{~d} y \wedge \mathrm{d} z-\left(\alpha \tilde{B}_{3}\right)_{x} \mathrm{~d} z \wedge \mathrm{d} x,
$$

this $\alpha$-effect term gives the required coupling from the $\tilde{B}_{3}$ field to the $\tilde{B}_{1}$ and $\tilde{B}_{2}$ components (and note that the other components $i=1,2$ of $\tilde{\mathcal{E}}_{i}=-\eta\langle\tilde{\star} \mathrm{d} \tilde{\star} \tilde{B}\rangle_{i}$ would not, since the average yields a quantity independent of $z$ ).

If we take all the magnetic field components to be independent of $z$ we gain the governing equations as

$$
\begin{aligned}
\partial_{t} \tilde{B}_{1} & =\left(\alpha \tilde{B}_{3}\right)_{y}-\eta D_{1}, \\
\partial_{t} \tilde{B}_{2} & =-\left(\alpha \tilde{B}_{3}\right)_{x}-\eta D_{2}, \\
\partial_{t} \tilde{B}_{3} & =\left(\tilde{B}_{1} \partial_{x}+\tilde{B}_{2} \partial_{y}\right) u^{3}-\eta D_{3} .
\end{aligned}
$$

where the terms $D_{i}$ are the remaining diffusion terms, as discussed further below. Several remarks are in order in relating our results to the usual theory of kinematic dynamos. First we have extracted the $\alpha$-effect generating $\tilde{B}_{1}$ and $\tilde{B}_{2}$ components from the $\tilde{B}_{3}$ component of the magnetic flux: these $\alpha$-effect terms are crucial because they give the feedback loop that enables an $\alpha \omega$-dynamo to function. We have not sought coupling terms that for example generate a $\tilde{B}_{3}$ component from a $\tilde{B}_{1}$ or $\tilde{B}_{2}$ component, but this is not really necessary as we are assuming an $\omega$-effect, the terms $\left(\tilde{B}_{1} \partial_{x}+\tilde{B}_{2} \partial_{y}\right) u^{3}$ in the $\tilde{B}_{3}$ equation (4.33), to be present already. Typically in an $\alpha \omega$-dynamo the $\alpha$-effect is relatively weak, giving components $\tilde{B}_{1}, \tilde{B}_{2} \ll \tilde{B}_{3}$ and so any additional $\alpha$ type couplings would be a subdominant effect; if this is not the case then one can develop the theory of $\alpha^{2}$ - or $\alpha^{2} \omega$-dynamos, in which case these couplings need to be quantified.

The key point of this approach, as recognised by earlier authors going back to Soward (1972), is that applying a pull back keeps the structure of the advection and stretching terms in (4.19) (those not involving $\eta$ ) and it is not necessary to average these: all the averaging is done in the diffusion term. Expanding the diffusion term allows an $\alpha$-effect to emerge that is not present in the original equations and that parameterises how fluctuations superposed on a background flow diffusively generate other magnetic flux components. Of course the identification of an $\alpha$-effect was a key contribution to dynamo theory, in this context in the seminal papers of Braginskv (1964a b) with parallel work by Parker, Steenbeck, Krause and Rädler as reviewed in Moffatt and Dormy (2019). In short, introducing the above $\alpha$-effect and ignoring other effects of the fluctuating flow leads to the classic induction equation augmented by the $\alpha$ term (4.29), as (4.314.33) or

$$
\partial_{t} \tilde{B}+\mathcal{L}_{\bar{u}} \tilde{B}=\mathrm{d}\left(\alpha \tilde{B}_{3} \mathrm{~d} z\right)-\eta \mathrm{d} \star \mathrm{d} \star \tilde{B}
$$

\subsection{General case}

Let us now return to the general setting of the induction equation

$$
\partial_{t} B+\mathcal{L}_{u} B=-\eta \mathrm{d} \delta B=\mathrm{d} \mathcal{E}, \quad \mathcal{E}=-\eta \delta B,
$$

with any metric $g$ and flow $u$, where $\mathcal{E}$ is again the 1-form emf. We apply the earlier decomposition $\phi=\xi \circ \bar{\phi}$ in (4.17) and apply a pull back $\xi^{*}$ to the equation so as to remove some fluctuating component of the flow - as usual we have in mind waves on a simpler background flow $\bar{u}$ generated by $\bar{\phi}$. The resulting equation is as in (4.19):

$$
\partial_{t} \tilde{B}+\mathcal{L}_{\bar{u}} \tilde{B}=\mathrm{d} \tilde{\mathcal{E}}
$$

Here as above $\tilde{\mathcal{E}}$ is the pulled-back emf given by

$$
\tilde{\mathcal{E}}=\xi^{*} \mathcal{E}=-\eta \xi^{*}(\delta B)=-\eta \xi^{*}\left[\delta\left(\xi_{*} \tilde{B}\right)\right]=-\eta \tilde{\delta} \tilde{B},
$$


where we recall that $\tilde{B}=\xi^{*} B$ is the pulled back field and where the second equality here defines the pullback $\tilde{\delta}=\xi^{*} \delta \xi_{*}=\tilde{\star} \mathrm{d} \tilde{\star}$ of the codifferential operator $\delta$.

We now follow this by averaging over a family $\xi^{\iota}$ of fluctuations to obtain

$$
\partial_{t} \bar{B}^{\mathrm{L}}+\mathcal{L}_{\bar{u}} \bar{B}^{\mathrm{L}}=\mathrm{d} \overline{\mathcal{E}}^{\mathrm{L}}, \quad \overline{\mathcal{E}}^{\mathrm{L}}=-\eta\langle\tilde{\delta} \tilde{B}\rangle=-\eta \overline{\delta B}^{\mathrm{L}},
$$

where

$$
\bar{B}^{\mathrm{L}}=\langle\tilde{B}\rangle=\left\langle\xi^{*} B\right\rangle .
$$

There is no approximation up to this point. This expression for the mean emf $\overline{\mathcal{E}}^{\mathrm{L}}$ involves both the magnetic field $\tilde{B}$ and the fluctuations through $\tilde{\delta}$ and in an exact development the product $\overline{\delta B}^{\mathrm{L}}$ generally cannot be broken up into the product of two averages. We therefore write $\tilde{B}$ as the sum of its (Lagrangian) mean and fluctuating component, setting

$$
\tilde{B}=\bar{B}^{\mathrm{L}}+\tilde{B}^{\ell}, \quad B=\xi_{*} \bar{B}^{\mathrm{L}}+B^{\ell}, \quad \tilde{B}^{\ell}=\xi^{*} B^{\ell},
$$

and writing

$$
\overline{\mathcal{E}}^{\mathrm{L}}=-\eta \bar{\delta}^{\mathrm{L}} \bar{B}^{\mathrm{L}}-\eta \overline{\delta \bar{B}^{\ell}}
$$

Of these two terms, the first, $-\eta \bar{\delta}^{\mathrm{L}} \bar{B}^{\mathrm{L}}$, represents a transport effect: the effect of the operator $\bar{\delta}^{\mathrm{L}}$ on a mean field $\bar{B}^{\mathrm{L}}$, as discussed informally in the previous section. Our aim is to obtain expressions for this operator, which includes an $\alpha$-effect and effective diffusivity. We will not consider the second term, $\eta{\overline{\delta B^{\ell}}}^{\mathrm{L}}$ as it would usually be handled separately in a dynamo calculation and be neglected at leading order. As in any transport problem, one calculates the coefficients or operators governing the transport of a mean field, and this is then fed into a more global calculation which involves further assumptions, such as scale separation, or a parameter being large or small, in order to neglect some terms. In the case of the Braginsky dynamo, a limit of large magnetic Reynolds number, here $\eta \ll 1$, results in the fluctuating fields $\tilde{B}^{\ell}$ being negligible, even for large Lagrangian displacements encoded in $\xi$ and so in $\bar{\delta}^{\mathrm{L}}$.

For these reasons we will neglect the second term in (4.41) and focus on the first, our aim being to elucidate the structure of the averaged operator $\bar{\delta}^{\mathrm{L}}$. To avoid introducing new quantities we will use $\doteq$ to signal that the second term in (4.41) has been dropped, and write

$$
\overline{\mathcal{E}}^{\mathrm{L}} \doteq-\eta \bar{\delta}^{\mathrm{L}} \bar{B}^{\mathrm{L}} .
$$

The operator $\bar{\delta}^{\mathrm{L}}=\langle\tilde{\delta}\rangle$ involves derivatives and we will expand this shortly as

$$
\left.\left.\overline{\mathcal{E}}^{\mathrm{L}} \doteq-\eta \bar{\delta}^{\mathrm{L}} \bar{B}^{\mathrm{L}}=\alpha\right\lrcorner \bar{B}^{\mathrm{L}}+\gamma\right\lrcorner \nabla \bar{B}^{\mathrm{L}}
$$

or, in coordinates,

$$
\overline{\mathcal{E}}_{i}^{\mathrm{L}} \doteq \alpha_{i}^{n p} \bar{B}_{n p}^{\mathrm{L}}+\gamma_{i}^{k n p} \nabla_{k} \bar{B}_{n p}^{\mathrm{L}} .
$$

Here the $\alpha_{i}^{n p}$ are the components of the $\alpha$ tensor, and the $\gamma_{i}^{k n p}$ are the components of the effective difusivity tensor. Our approach below will be to work first with the pull back $\tilde{\mathcal{E}}=\tilde{\delta} \tilde{B}$ and then ensemble average: at that point we can replace $\tilde{B}$ with $\bar{B}^{\mathrm{L}}$ and extract the $\alpha$-effect and $\gamma$ tensor.

We now derive an explicit expression for $\tilde{\mathcal{E}}$ in a way that sidesteps much of the coordinate computations of section 4.1 This relies on two observations. First, we use the relation between the codifferential $\delta$ and the covariant derivative $\nabla$ of differential forms (Frankel, 1997, Theorem 14.15) which, when applied to the 2 -form $B$, gives

$$
(\delta B)_{i}=g^{j k} \nabla_{k} B_{i j} .
$$

For completeness we establish this property in Appendix A, It makes it possible to rewrite $\tilde{\mathcal{E}}$ in (4.37) as

$$
\tilde{\mathcal{E}}_{i}=-\eta \tilde{g}^{j k} \tilde{\nabla}_{k} \tilde{B}_{i j}
$$


where we have introduced the pull back $\tilde{\nabla}=\xi^{*} \nabla$ of the covariant derivative $\nabla$. This is defined in a coordinate-free way by

$$
\tilde{\nabla}_{u} \tau \equiv\left(\xi^{*} \nabla\right)_{u} \tau=\xi^{*}\left[\nabla_{\xi_{*} u}\left(\xi_{*} \tau\right)\right]
$$

(Stein, 2017). In other words on the right-hand side we push $u$ and $\tau$ forwards, apply $\nabla$ and then pull the result back. This gives $\tilde{\nabla}$ as the covariant derivative with respect to the pulled back metric $\tilde{g}$. Rewriting (4.46) as

$$
\tilde{\mathcal{E}}_{i}=-\eta \tilde{g}^{j k}\left(\tilde{\nabla}_{k}-\nabla_{k}\right) \tilde{B}_{i j}-\eta \tilde{g}^{j k} \nabla_{k} \tilde{B}_{i j},
$$

we make the second observation: the difference of covariant derivatives, applied to any tensor, just returns a tensor (no derivatives are involved) involving connection coefficients $C_{j k}^{i}$ (symmetric in $j$ and $k$ ) with, for example,

$$
\left(\tilde{\nabla}_{k}-\nabla_{k}\right) v^{j}=C_{l k}^{j} v^{l}, \quad\left(\tilde{\nabla}_{k}-\nabla_{k}\right) \lambda_{j}=-C_{j k}^{l} \lambda_{l}
$$

for a vector field $v$ and a 1 -form field $\sigma$ (Stein, 2017). Here the coefficients $C_{j k}^{i}$ are the difference between the Christoffel symbols for the two metrics $\tilde{g}$ and $g$,

$$
C_{j k}^{i}=\tilde{\Gamma}_{j k}^{i}-\Gamma_{j k}^{i} .
$$

Using these and noting that

$$
\left(\tilde{\nabla}_{k}-\nabla_{k}\right) \tilde{B}_{i j}=-C_{k i}^{s} \tilde{B}_{s j}-C_{k j}^{s} \tilde{B}_{i s}
$$

we write

$$
\tilde{\mathcal{E}}_{i}=\eta \tilde{g}^{j k}\left(C_{k i}^{s} \tilde{B}_{s j}+C_{k j}^{s} \tilde{B}_{i s}-\tilde{g}^{p k} \nabla_{k} \tilde{B}_{i p}\right) .
$$

Bearing in mind (4.44) and the discussion there, we now replace $\tilde{B}$ by the mean field $\bar{B}^{\mathrm{L}}$ and ensemble average, meaning we only average over the metric $\tilde{g}$ and the connection coefficients $C_{j k}^{i}$ and not the field, to identify the tensors $\alpha$ and $\gamma$ as

$$
\begin{aligned}
\alpha_{i}^{n p} & =\eta\left\langle C_{k i}^{[n} \tilde{g}^{p] k}+\tilde{g}^{j k} \delta_{i}^{[n} C_{j k}^{p]}\right\rangle \\
\gamma_{i}^{k n p} & =-\eta \delta_{i}^{[n}\left\langle\tilde{g}^{p] k}\right\rangle
\end{aligned}
$$

(the $[n p]$ antisymmetrisation is optional as the $n p$ indices are to be contracted against the 2-form magnetic field). This gives an equation for the $\alpha$ tensor as derived from the tensor $C_{j k}^{i}$ giving the difference between the connection coefficients of the covariant derivative and the pulled back covariant derivative. This fundamental geometric formulation of $\alpha$ is new as far as we are aware.

We stress that formula (4.53) for the general tensor components $\alpha_{i}^{n p}$ is exact and covers finite amplitude fluctuations. It includes compressible or incompressible flow, this being the principal benefit of working with the magnetic flux 2-form $B$ rather than the vector field $b$ here. In appendix B we confirm that (4.53) gives the correct $\alpha$-effect for the case of a simple helical wave. In appendix $[$ we relate our approach to some wellestablished formulae in the literature, for example given in Moffatt and Dormv (2019). We start by stating an alternative, more classical expression for $\alpha_{i}^{n p}$, given as (C.2), which holds with the same generality as (4.53). We verify the equivalence of this expression with (4.53) and use it to recover the Euclidean results of Roberts and Soward (2006a,b).

\subsection{Perturbative calculation of the $\alpha$-effect}

All the above discussion is for finite amplitude maps $\xi$ and requires no small parameter in order to write down formulae for the $\alpha$-effect and effective diffusivity. In a realistic application the waves or fluctuations encoded in $\xi$ would be developed in powers of a small parameter $\varepsilon \ll 1$ as far as needed to obtain the leading non-zero $\alpha$-effect, together with the limit of small diffusivity, here $\eta \ll 1$, in the full dynamo calculation (Soward and Roberts, 2014). We can take two approaches to calculate $\alpha$ perturbatively for small amplitude $\varepsilon \ll 1$. The first $(\S 4.3 .1)$ is to take the pulled-back codifferential $\tilde{\delta}=\tilde{\star} \mathrm{d} \tilde{\star}$, and to expand the pulled-back 
Hodge star operator $\tilde{\star}=\xi^{*} \star$ as a power series in the amplitude of the fluctuations, this involving the Lie derivative of $\star$ in a vector field $q$ used to generate the fluctuation map $\xi$ at any time. The second approach (\$4.3.2) works from (4.48), which employs a pulled-back covariant derivative $\tilde{\nabla}$ in the difference $\left(\tilde{\nabla}_{k}-\nabla_{k}\right) \tilde{B}_{i j}$. This difference of derivatives, which relates to the conection coefficient tensor $C_{j k}^{i}$, can likewise be expanded as a power series in the amplitude of the fluctuating map $\xi$. These two approaches give different but equivalent formulae for the $\alpha$-effect corresponding to perturbative expansions of (C.2) and (4.53), respectively.

\subsubsection{Perturbative calculation based on $\delta$}

Let us return to the more abstract setting, and write the emf as

$$
\left.\left.\overline{\mathcal{E}}^{\mathrm{L}} \doteq-\eta \bar{\delta}^{\mathrm{L}} \bar{B}^{\mathrm{L}}=-\eta\langle\tilde{\delta}\rangle \bar{B}^{\mathrm{L}}=-\eta\langle\tilde{\star} \mathrm{d} \tilde{\star}\rangle \bar{B}^{\mathrm{L}}=\alpha\right\lrcorner \bar{B}^{\mathrm{L}}+\gamma\right\lrcorner \nabla \bar{B}^{\mathrm{L}} .
$$

Here the derivatives in $\langle\delta\rangle$ and the $\langle\star d \star\rangle$ act on quantities to their right, including the mean magnetic field $\bar{B}^{\mathrm{L}}$. We can develop $\xi(x)$ as a perturbation series, writing

$$
\xi^{i}(x)=x^{i}+\varepsilon q^{i}(x)+\frac{1}{2} \varepsilon^{2} q^{j}(x) \partial_{j} q^{i}(x)+\cdots
$$

(we suppress the dependence of all these quantities on time $t$ ). Here $q$ is a vector field defined on $\mathcal{M}$ (generally depending on time) which, when integrated over a fictitious time variable $s$ from 'time' $s=0$ to $s=\varepsilon$ (at any fixed time $t$ ), effects the map $\xi$ (Soward and Roberts, 2010; Gilbert and Vanneste, 2018), or formally $\xi=\exp (\varepsilon q)$. For simplicity we take $q$ to depend on $(x, t)$ but not on $s$ : it is steady in fictitious time. At leading order a pull back is then given for any tensor $\tau$ by

$$
\xi^{*} \tau=\tau+\varepsilon \mathcal{L}_{q} \tau+\frac{1}{2} \varepsilon^{2} \mathcal{L}_{q} \mathcal{L}_{q} \tau+\cdots,
$$

from the definition of the Lie derivative. Now suppose we expand (4.55) in powers of $\varepsilon$ to obtain

$$
\begin{aligned}
\overline{\mathcal{E}}^{\mathrm{L}} \doteq-\eta\langle\tilde{\star} \mathrm{d} \tilde{\star}\rangle \bar{B}^{\mathrm{L}} & =-\eta\left[\star \mathrm{d} \star+\varepsilon\left\langle\mathcal{L}_{q} \star\right\rangle \mathrm{d} \star+\varepsilon \star \mathrm{d}\left\langle\mathcal{L}_{q} \star\right\rangle\right. \\
& \left.+\frac{1}{2} \varepsilon^{2}\left\langle\mathcal{L}_{q} \mathcal{L}_{q} \star\right\rangle \mathrm{d} \star+\varepsilon^{2}\left\langle\mathcal{L}_{q} \star \mathrm{d} \mathcal{L}_{q^{\star}}\right\rangle+\frac{1}{2} \varepsilon^{2} \star \mathrm{d}\left\langle\mathcal{L}_{q} \mathcal{L}_{q} \star\right\rangle+\cdots\right] \bar{B}^{\mathrm{L}},
\end{aligned}
$$

where we can think of the star operator as simply the tensor with components $\star_{i}^{k l}=\frac{1}{2} g_{i j} \mu^{j k l}$ giving the map from 2-forms to 1-forms in this context; see (4.21).

There is a proliferation of terms in (4.58); however in typical applications many will be zero by virtue of the symmetry of the underlying system. For example in our sketch $\alpha \omega$-dynamo we restricted to a metric with a simple structure (4.4), independent of the third coordinate, and extracted an $\alpha$-effect taking $B_{3}$ field to $\mathcal{E}_{3}$, needed to close the dynamo loop. In a 'working' dynamo model specific choices would need to be made, and if the fluctuations encoded in $q$ lead to averages such as $\left\langle\mathcal{L}_{q} \star\right\rangle$ and $\left\langle\mathcal{L}_{q} \mathcal{L}_{q} \star\right\rangle$ retaining a simple structure then the terms involving these averages above may well be zero, or not contribute to the components of the $\alpha$ tensor needed in that dynamo. For example in the informal approach of section 4.1, the key term is (4.29) and involves an average with the exterior derivative $d$ sandwiched between two quantities. Bearing in mind that this would need to be checked on a case-by-case basis, we will calculate just the key term involving $d$ similarly sandwiched inside the average, writing

$$
\overline{\mathcal{E}}^{\mathrm{L}} \doteq-\eta \varepsilon^{2}\left\langle\mathcal{L}_{q} \star \mathrm{d} \mathcal{L}_{q} \star\right\rangle \bar{B}^{\mathrm{L}} .
$$

To calculate $\mathcal{L}_{q} \star$ we follow the development in Trautman (1984). Define the tensor $h$ by

$$
\mathcal{L}_{q} g=h g \quad \text { or } \quad\left(\mathcal{L}_{q} g\right)_{i j}=h_{i}^{k} g_{k j} .
$$

Then we have

$$
\mathcal{L}_{q} \mu^{i j k}=-\frac{1}{2}(\operatorname{tr} h) \mu^{i j k}, \quad \operatorname{tr} h=h_{i}^{i}
$$


and bearing in mind that $\star_{i}^{k l}=\frac{1}{2} g_{i j} \mu^{j k l}$, it follows that

$$
\mathcal{L}_{q} \star=\left(h-\frac{1}{2} \operatorname{tr} h\right) \star \quad \text { or } \quad\left(\mathcal{L}_{q} \star\right)_{i}^{j k}=\left(h_{i}^{l}-\frac{1}{2} \delta_{i}^{l} \operatorname{tr} h\right) \frac{1}{2} g_{l m} \mu^{m j k} .
$$

From (4.60), the tensor $h$ is plainly linked to the deformation tensor $\sigma$, that is the rate of change (Lie derivative) of the metric in the flow $q$, with

$$
\sigma \equiv h g=\mathcal{L}_{q} g=\nabla q_{\mathrm{b}}+\left(\nabla q_{\mathrm{b}}\right)^{\mathrm{T}} \quad \text { or } \quad \sigma_{i j} \equiv h_{i}^{k} g_{k j}=\left(\mathcal{L}_{q} g\right)_{i j}=\nabla_{i} q_{j}+\nabla_{j} q_{i}
$$

We also need the fact that in the definition of $d$ acting on a 1-form in (4.22) partial derivatives may be replaced by covariant derivatives (given that there is no torsion for a covariant derivative induced by a metric), and so we have for any 1 -form $\lambda$

$$
(\mathrm{d} \lambda)_{i j}=2 \nabla_{[i} \lambda_{j]}=\nabla_{i} \lambda_{j}-\nabla_{j} \lambda_{i}
$$

With these definitions, the $\alpha$ tensor can be written in a variety of ways,

$$
\begin{aligned}
\alpha_{i}^{n p} & =-\eta \varepsilon^{2} g_{r j} \mu^{j k l} g_{s m} \frac{1}{2} \mu^{m n p}\left\langle\left(h_{i}^{r}-\frac{1}{2} \delta_{i}^{r} \operatorname{tr} h\right) \nabla_{k}\left(h_{l}^{s}-\frac{1}{2} \delta_{l}^{s} \operatorname{tr} h\right)\right\rangle \\
& =-\eta \varepsilon^{2} \mu^{j k l} \frac{1}{2} \mu^{m n p}\left\langle\left(\sigma_{i j}-\frac{1}{2} g_{i j} \operatorname{tr} \sigma\right) \nabla_{k}\left(\sigma_{l m}-\frac{1}{2} g_{l m} \operatorname{tr} \sigma\right)\right\rangle \\
& =-\eta \varepsilon^{2} \mu^{j k l} \frac{1}{2} \mu^{m n p}\left\langle\left(\nabla_{i} q_{j}+\nabla_{j} q_{i}-g_{i j} \operatorname{div} q\right) \nabla_{k}\left(\nabla_{l} q_{m}+\nabla_{m} q_{l}-g_{l m} \operatorname{div} q\right)\right\rangle,
\end{aligned}
$$

with $\operatorname{tr} \sigma=\sigma_{i j} g^{i j}=2 \operatorname{div} q=2 \nabla_{i} q^{i}$. Thus the $\alpha$-effect is expressed in a general form either in terms of the deformation tensor $\sigma$ or the vector field $q$ (and the corresponding 1-form $q_{b}$ ) generating the fluctuations and so the family of maps $\xi$. Note that if all maps are volume-preserving and flows incompressible we have $\operatorname{tr} \sigma=0$ and then we can write $\alpha$ compactly as

$$
\alpha_{i}^{n p}=-\eta \varepsilon^{2} \mu^{j k l} \frac{1}{2} \mu^{m n p}\left\langle\sigma_{i j} \nabla_{k} \sigma_{l m}\right\rangle, \quad(\operatorname{tr} \sigma=0) .
$$

There do not seem to be many general simplifications beyond this point, except to note that the term $\mu^{j k l} \nabla_{k} \nabla_{l} q_{m}$ in (4.67) involves the Riemann tensor and so vanishes if $\mathcal{M}$ is flat.

\subsubsection{Perturbative calculation based on $\nabla$}

An alternative approach is to apply techniques in Stein (2017) to write down forms of the $\alpha$ tensor based on (4.48), namely,

$$
\alpha_{i}^{n p} \bar{B}_{n p}^{\mathrm{L}}=-\eta\left\langle\tilde{g}^{j k}\left(\tilde{\nabla}_{k}-\nabla_{k}\right)\right\rangle \bar{B}_{i j}^{\mathrm{L}} .
$$

Since the averaged field $\bar{B}^{\mathrm{L}}$ acts here as just a test field on which to do calculations, we replace it in this section only by $B$, to lighten notation:

$$
\alpha_{i}^{n p} B_{n p}=-\eta\left\langle\tilde{g}^{j k}\left(\tilde{\nabla}_{k}-\nabla_{k}\right)\right\rangle B_{i j} .
$$

We introduce a series expansion for the $\alpha$ tensor,

$$
\alpha_{i}^{n p}=\varepsilon \alpha_{(1) i}^{n p}+\varepsilon^{2} \alpha_{(2) i}^{n p}+\cdots,
$$

with

$$
\alpha_{(m) i}^{n p} B_{n p}=-\left.\frac{\eta}{m !} \frac{\mathrm{d}^{m}}{\mathrm{~d} s^{m}}\right|_{s=0}\left\langle\left(\xi^{*} g^{j k}\right) \xi^{*}\left(\nabla_{k} \xi_{*} B_{i j}\right)-\left(\xi^{*} g^{j k}\right) \nabla_{k} B_{i j}\right\rangle .
$$

Here, as above we suppose that the map $\xi$ is effected by a vector field $q$ over an interval of fictitious time $0 \leq s \leq \varepsilon ; q$ generally depends on time but not on $s$. We have for any tensor $\tau$,

$$
\mathcal{L}_{q} \xi^{*} \tau=\frac{\mathrm{d}}{\mathrm{d} s} \xi^{*} \tau, \quad \mathcal{L}_{-q} \xi_{*} \tau=\frac{\mathrm{d}}{\mathrm{d} s} \xi_{*} \tau .
$$


Using these with (4.72) for $m=1$ and $m=2$, and averaging with $\langle\cdot\rangle$ gives after some algebra,

$$
\begin{aligned}
& \alpha_{(1) i}^{n p} B_{n p}=-\eta g^{j k}\left[\left\langle\mathcal{L}_{q}\right\rangle, \nabla_{k}\right] B_{i j}, \\
& \alpha_{(2) i}^{n p} B_{n p}=-\eta\left\langle\left(\mathcal{L}_{q} g^{j k}\right)\left[\mathcal{L}_{q}, \nabla_{k}\right]\right\rangle B_{i j}-\eta \frac{1}{2} g^{j k}\left\langle\left[\mathcal{L}_{q},\left[\mathcal{L}_{q}, \nabla_{k}\right]\right]\right\rangle B_{i j},
\end{aligned}
$$

where the square brackets denote a commutator, for example $\left[\mathcal{L}_{q}, \nabla_{k}\right] B_{i j}=\mathcal{L}_{q} \nabla_{k} B_{i j}-\nabla_{k} \mathcal{L}_{q} B_{i j}$. All the terms in (4.58) are included here though, as mentioned above, in typical applications one would have $\alpha_{(1) i}^{n p}=0$ and many terms vanishing from $\alpha_{(2) i}^{n p}$.

\subsection{Summary}

We summarise the key results of section 4: we recall that these are stated using the magnetic field as a 2 -form, and that the tilde annotation denotes any pulled-back quantity, that is transported from the flow with fluctuations or waves, to the background flow. The first, exact result is (4.48, 4.53), which express the $\alpha$-effect

$$
\alpha_{i}^{n p} \bar{B}_{n p}^{\mathrm{L}}=-\eta\left\langle\tilde{g}^{j k}\left(\tilde{\nabla}_{k}-\nabla_{k}\right)\right\rangle \bar{B}_{i j}^{\mathrm{L}}=\eta\left\langle\tilde{g}^{j k} C_{k i}^{s}\right\rangle \bar{B}_{s j}^{\mathrm{L}}+\eta\left\langle\tilde{g}^{j k} C_{k j}^{s}\right\rangle \bar{B}_{i s}^{\mathrm{L}},
$$

in terms of the difference between the covariant derivatives $\tilde{\nabla}$ and $\nabla$, or in terms of the connection coefficients $C_{j k}^{i}$, the difference in the connections between the metric $g$ and the pulled-back metric $\tilde{g}$. This formula can be applied in space of any number of dimensions; an alternative expression for $\alpha_{i}^{n p}$, more closely related to earlier literature but restricted to three dimensions, is given in (C.2).

We give a variety of approximate formulae for the $\alpha$ tensor in sections 4.3 .1 and 4.3 .2 using a smallamplitude expansion in the magnitude $\varepsilon$ of the fluctuations. In particular (4.66),

$$
\alpha_{i}^{n p} \bar{B}_{n p}^{\mathrm{L}}=-\eta \varepsilon^{2} \mu^{j k l} \frac{1}{2} \mu^{m n p}\left\langle\left(\sigma_{i j}-\frac{1}{2} g_{i j} \operatorname{tr} \sigma\right) \nabla_{k}\left(\sigma_{l m}-\frac{1}{2} g_{l m} \operatorname{tr} \sigma\right)\right\rangle \bar{B}_{n p}^{\mathrm{L}},
$$

expresses the $\alpha$-effect in terms of the deformation tensor $\sigma=\mathcal{L}_{q} g$, that is the Lie derivative of the metric in the flow $q$ generating the fluctuations.

Note that we have preferred to work with the closed magnetic 2 -form $B$ with $\mathrm{d} B=0$ rather than the solenoidal magnetic vector field $b$ with $\operatorname{div} b=0$, noting that $b\lrcorner \mu=B$ and $\mathrm{d}(b\lrcorner \mu)=\mu \operatorname{div} b$. For the general case of compressible flow $\mathrm{d} B=0$ translates into $\mathrm{d} \tilde{B}=0$ and so a solenoidal mean field $\mathrm{d} \bar{B}^{\mathrm{L}}=0$. However in terms of $b$ we only obtain that $\mathrm{d}\left(\overline{b\lrcorner \mu}^{\mathrm{L}}\right)=0$ and so generally $\operatorname{div} \bar{b}^{\mathrm{L}} \neq 0$, in other words $\left.\mathrm{d}\left(\bar{b}^{\mathrm{L}}\right\lrcorner \mu\right) \neq 0$ : the Lagrangian average of $b$ is not solenoidal. This could be cured by recognising that $b$ transforms as a tensor of weight -1 , but that is equivalent to using $B$ in any case. If all flows and maps are volume preserving, $\mu=\tilde{\mu}=\bar{\mu}^{\mathrm{L}}$, this issue evaporates, $\operatorname{div} \bar{b}^{\mathrm{L}}=0$ and in the calculations above we can easily express the $\alpha$-effect in terms of the mean magnetic vector field $\bar{b}^{\mathrm{L}}$ by replacing

$$
\frac{1}{2} \mu^{m n p} \bar{B}_{n p}^{\mathrm{L}}=\left(\bar{b}^{\mathrm{L}}\right)^{m},
$$

in equations such as (4.77) above.

\section{Concluding remarks}

In this paper, we have looked at MHD from a geometric perspective, both to derive the governing equations and their properties, and to revisit some basic applications, to Alfvén waves on a uniform field and to the analysis of the Braginsky dynamo. There are many attractions of a geometric approach. In concrete terms, results are valid for any coordinate system, and so no changes need to be made when going from, say, Cartesian coordinates to cylindrical polar coordinates, whereas otherwise this requires special consideration (Soward and Roberts, 2014). Furthermore results remain correct even when one might find it helpful to adopt a non-orthogonal coordinate system, for example to use a buoyancy or pressure coordinate instead 
of a vertical coordinate in a geophysical or astrophysical setting. This flexibility is convenient even when working in $\mathbb{R}^{3}$; in fact there is no difference in the theoretical development up to the point where second derivatives come in and curvature plays a role through the Riemann tensor, for example in a diffusion operator (Gilbert, Riedinger and Thuburn, 2014). For theoretical developments, the machinery of pull backs and push forwards allows one to apply mappings to equations while preserving their structure as much as possible. Essentially, a neighbourhood of any point in the interior of $\mathcal{M}$ is much like any other point from the viewpoint of the basic operations $\mathcal{L}, \mathrm{d}$,$\lrcorner of differential geometry: we can take a calculation performed at one point and$ move it to another, provided we take the fields and all the necessary extra structure, that is the metric $g$ and volume form $\mu$, with us. This fact makes it possible to pin down why certain finite-amplitude approximations work, even if in real applications perturbation theory may well be needed for concrete calculations.

There are some disadvantages; for example we have defined $B, b$ and $\beta$, which are all the magnetic field in one version or another! To move between these we use the metric and/or volume form, and this allows the careful tracking of how quantities transform under mappings, pull backs or push forwards, applied to equations written in the form (2.8) and (2.41). Note that using the 'general relativity' notation (2.48, 2.49), while it introduces fewer quantities, hides the underlying differential geometric structure, and applying a pull back risks becoming entangled in transformations of Christoffel symbols, something generally worth avoiding.

Concerning Alfvén waves, we addressed only the most fundamental model, and we plan to look into compressible waves, and waves on non-uniform fields, to see how these can be parameterised within the present framework, in parallel with similar developments in the literature of geophysical fluid dynamics; see, for example, Bühler (2009). One important point to note, as discussed in more detail in Gilbert and Vanneste (2018), and which emerges in other studies such as Soward and Roberts (2010), is that given a family of flows with waves, there is no imperative need to define what the 'mean flow' is at the outset. Although we use a bar (e.g. $\bar{u}, \bar{b}$ ), we refer to such fields as background fields. In the decomposition (3.7) which underlies all the work using pull backs, or equivalently hybrid Euler-Lagrangian coordinates or GLM-type theories, the choice of the mean map $\bar{\phi}$ and flow $\bar{u}$ is open, free to choose depending on applications. In our development, no assumption has been made along the lines that some sort of average of the fluctuations is zero, and in fact such assumptions are not easy to deploy in a general setting. Several possible choices of a mean flow can be made, generally, and correspond to different divisions between mean flow and fluctuations, typically important at quadratic order in fluctuation amplitude. The issues are discussed in Soward and Roberts (2010) and Gilbert and Vanneste (2018), with references to related literature.

For the Braginsky dynamo, we gave a sketch of the classic dynamo set-up in a general geometry. Here there is a background flow giving an $\omega$-effect, converting transverse field components $B_{1}, B_{2}$ to azimuthal field $B_{3}$, but there is no feedback from the background geometry or flow that can regenerate the transverse field, even with diffusion $\eta>0$. In short, we restricted attention to a limited, but still wide, family of possible metrics $g(x, y)$ and background flows $\bar{u}(x, y)$. We then introduced some waves or fluctuations, giving $z$-dependence by means of a Lagrangian map $\xi$ and showed how the pull back of the equations under $\xi$ preserves the structure of the ideal terms, while the diffusion term, present for $\eta>0$, can be understood in terms of the pull back of the codifferential operator $\delta$. Averaging over a family of such waves, we then obtained the $\alpha$-effect, written in various forms in (4.53), (4.65) 4.68) and (4.74, 4.75, (C.2), that can close the dynamo loop and lead to a sustained or growing field.

We stress that our study is a sketch, aiming at exposing the geometry behind the origin and definition of the $\alpha$-tensor. Nonetheless the approach using the compact notation and identities of differential geometry allows us to interpret the $\alpha$-effect in terms of pull backs and connections, derive established formulae and give new expressions, all valid for arbitrary coordinate systems. We note though that writing down formulae for the $\alpha$ tensor is just one element of the bigger picture, which requires the scaling of field component magnitudes in terms of the magnetic Reynolds number, setting up a suitable eigenvalue problem, and keeping track of the order of the errors involved. However this is already well studied in the literature and we refer the reader to Soward and Roberts (2014) for detailed discussion. 


\section{Acknowledgements}

ADG is very grateful to the Leverhulme Trust for the award of a Research Fellowship, which enabled the continuing collaboration with JV and the work presented in this paper. AG also benefitted from STFC funding, research grant ST/R000891/1. We are grateful to Darryl Holm, Marcel Oliver and Andrew Soward for many useful discussions, and to the referees for constructive criticisms and additional references. We thank Leo Stein for the use of his notes in Stein (2017).

\section{A Derivation of (4.45)}

We establish (4.45) in three dimensions, using the coordinate expression

$$
(\star B)_{i}=\frac{1}{2} g_{i j} \mu^{j k l} B_{k l}
$$

for the Hodge $\star$ operator applied to a 2 -form $B$ (see (4.21)). Noting (4.64), we have

$$
\begin{aligned}
(\delta B)_{i}=(\star \mathrm{d} \star B)_{i} & =g_{i j} \mu^{j k l} \nabla_{k}\left(g_{l m} \frac{1}{2} \mu^{m n p} B_{n p}\right), \\
& =g_{i j} \mu^{j k l} g_{l m} \frac{1}{2} \mu^{m n p} \nabla_{k} B_{n p} \\
& =g^{p k} \nabla_{k} B_{i p},
\end{aligned}
$$

using that

$$
\mu^{j k l} g_{l m} \mu^{m n p}=g^{j n} g^{p k}-g^{j p} g^{n k}=2 g^{j[n} g^{p] k},
$$

to obtain the last line. This gives $\delta B$ as minus the divergence of the 2 -form $B$ as defined by Frankel (1997).

\section{B The $\alpha$-effect from helical waves}

As a basic example of the calculation of $\alpha$ in sections 4.1 and 4.2, and to link with well-established theory, suppose we take a situation similar to that in section 3.3. with $g=\mathrm{d} x^{2}+\mathrm{d} y^{2}+\mathrm{d} z^{2}$, and some waves given by Lagrangian displacements of the form

$$
\xi(x, y, z, t)=(x+F(z-c t), y+H(z-c t), z) .
$$

Using (3.40), the pulled back metric and its inverse are

$$
\tilde{g}_{i j}=\left(\begin{array}{ccc}
1 & 0 & F^{\prime} \\
0 & 1 & H^{\prime} \\
F^{\prime} & H^{\prime} & 1+F^{\prime 2}+H^{\prime 2}
\end{array}\right), \quad \tilde{g}^{i j}=\left(\begin{array}{ccc}
1+F^{2} & H^{\prime} F^{\prime} & -F^{\prime} \\
H^{\prime} F^{\prime} & 1+H^{\prime 2} & -H^{\prime} \\
-F^{\prime} & -H^{\prime} & 1
\end{array}\right)
$$

and have determinant 1 so that $\tilde{\varsigma}=1$ also (the map $\xi$ is volume-preserving). Here we find from (4.29) that

$$
\alpha=\eta\left\langle\tilde{g}_{31} \partial_{z} \tilde{g}_{23}-\tilde{g}_{32} \partial_{z} \tilde{g}_{13}\right\rangle=\eta\left\langle F^{\prime} H^{\prime \prime}-H^{\prime} F^{\prime \prime}\right\rangle .
$$

Thus for example if we take a case with no background flow $\bar{u}=0$ and a wave with

$$
u=w=U \sin (k(z-c t)) \partial_{x}+U \cos (k(z-c t)) \partial_{y},
$$

then we have the fluctuating map given by

$$
F(s)=U \omega^{-1} \cos k s, \quad H(s)=-U \omega^{-1} \sin k s, \quad c \equiv \omega / k,
$$

and

$$
\alpha=-\eta U^{2} k^{3} \omega^{-2} .
$$


Note that the (kinetic) helicity of the wave is given by the integral of the helicity form defined by

$$
h_{\mathrm{K}}=\nu \wedge \mathrm{d} \nu=-k^{-2} \omega^{2}\left\langle F^{\prime} H^{\prime \prime}-H^{\prime} F^{\prime \prime}\right\rangle \mathrm{d} x \wedge \mathrm{d} y \wedge \mathrm{d} z=U^{2} k \mathrm{~d} x \wedge \mathrm{d} y \wedge \mathrm{d} z
$$

and so here positive helicity gives rise to a negative cofficient, $\alpha=-\eta k^{2} \omega^{-2} h_{\mathrm{K}}$; this is in keeping with the traditional sign convention in the literature. In this example there is no background flow and so the displacements given by $\xi$ are easily related to the flow field, with $u=w$. If there is a background flow then this needs to be taken into account via (3.11).

As a check it is interesting to calculate the $\alpha$ tensor components from (4.53). The Christoffel symbols $\Gamma_{j k}^{i}$ for $g$ are all zero and so we compute the $\tilde{\Gamma}_{j k}^{i}$ for $\tilde{g}$. We have then using standard notation

$$
\tilde{\Gamma}_{133}=F^{\prime \prime}, \quad \tilde{\Gamma}_{233}=H^{\prime \prime}, \quad \tilde{\Gamma}_{333}=F^{\prime} F^{\prime \prime}+H^{\prime} H^{\prime \prime}, \quad C_{33}^{1}=\tilde{\Gamma}_{33}^{1}=F^{\prime \prime}, \quad C_{33}^{2}=\tilde{\Gamma}_{33}^{2}=H^{\prime \prime},
$$

other terms being zero. This gives the only non-zero terms for $\alpha_{i}^{j k}$ as $\alpha_{3}^{12}=-\alpha_{3}^{21}=\frac{1}{2} \alpha$ with $\alpha$ given in (B.6). Referring to the calculations of section 4.3.1, in this example $\varepsilon q^{1}=\varepsilon q_{1}=F, \varepsilon q^{2}=\varepsilon q_{2}=H$ and there is agreement between the formulae for the $\alpha$ tensor here and in that section.

\section{Explicit calculations of the $\alpha$-effect}

In this appendix we relate our calculations of the $\alpha$-effect to formulae found in the literature. To do so, we first give an expression for the $\alpha$ tensor alternative to (4.53) and closer to those found elsewhere. This formula is obtained from (4.25) rewritten as

$$
\tilde{\mathcal{E}}_{i}=-\eta \tilde{g}_{i j} \tilde{\mu}^{j k l} \nabla_{k}\left(\tilde{g}_{l m} \frac{1}{2} \tilde{\mu}^{m n p} \tilde{B}_{n p}\right),
$$

making the effect of the pull back on the metric and volume form explicit. Here we have used the pulled back volume form $\tilde{\mu}=\tilde{\varsigma}^{-1} \mathrm{~d} x^{1} \wedge \mathrm{d} x^{2} \wedge \mathrm{d} x^{3}$ with covariant components $\tilde{\mu}_{i j k}=\tilde{\varsigma}^{-1} \varepsilon_{i j k}$ and the corresponding contravariant tensor $\tilde{\mu}^{i j k}=\tilde{\varsigma} \varepsilon^{i j k}$, together with (4.64). Comparing (C.1) with (4.52) gives the alternative to (4.53 4.54),

$$
\begin{aligned}
\alpha_{i}^{n p} & =-\eta\left\langle\frac{1}{2} \tilde{g}_{i j} \tilde{\mu}^{j k l} \nabla_{k}\left(\tilde{g}_{l m} \tilde{\mu}^{m n p}\right)\right\rangle, \\
\gamma_{i}^{k n p} & =-\eta\left\langle\frac{1}{2} \tilde{g}_{i j} \tilde{\mu}^{j k l} \tilde{g}_{l m} \tilde{\mu}^{m n p}\right\rangle,
\end{aligned}
$$

It is instructive to show that (C.2 C.3) are equivalent to (4.53, 4.54) in a direct manner. For $\gamma$, this is immediate using (A.5). For $\alpha$, we compute

$$
\begin{aligned}
-\tilde{g}_{i j} \tilde{\mu}^{j k l} \nabla_{k}\left(\tilde{g}_{l m} \tilde{\mu}^{m n p}\right) & =\tilde{g}_{i j} \tilde{\mu}^{j k l}\left(\tilde{\nabla}_{k}-\nabla_{k}\right)\left(\tilde{g}_{l m} \tilde{\mu}^{m n p}\right) \\
& =\tilde{g}_{i j} \tilde{\mu}^{j k l}\left(C_{k s}^{n} \tilde{g}_{l m} \tilde{\mu}^{m s p}+C_{k s}^{p} \tilde{g}_{l m} \tilde{\mu}^{m n s}-C_{k l}^{s} \tilde{g}_{s m} \tilde{\mu}^{m n p}\right),
\end{aligned}
$$

using that $\tilde{\nabla} \tilde{g}=0$ and $\tilde{\nabla} \tilde{\mu}=0$ and (4.49). The last term vanishes by (anti)symmetry. From (A.5) we then find

$$
-\tilde{g}_{i j} \tilde{\mu}^{j k l} \nabla_{k}\left(\tilde{g}_{l m} \tilde{\mu}^{m n p}\right)=\tilde{g}^{k p} C_{k i}^{n}-\tilde{g}^{k s} C_{k s}^{n} \delta_{i}^{p}+\tilde{g}^{k s} C_{k s}^{p} \delta_{i}^{n}-C_{k i}^{p} \tilde{g}^{k n}=2 \tilde{g}^{k[p} C_{k i}^{n]}+2 \tilde{g}^{k s} C_{k s}^{[p} \delta_{i}^{n]},
$$

and using this in (C.2) recovers (4.53), as expected.

We now turn to concrete calculations, recalling that in each realisation of one of our flows, the fluctuating map is $x \rightarrow \xi(x)$, so if the point with coordinates $x^{i}$ marks a Lagrangian parcel in the background flow at some time, $\xi^{i}(x)$ are its coordinates in the full flow. The pull back from $\xi(x)$ to $x$ for vectors and 1-forms is simply the Cauchy solution, namely

$$
\tilde{v}(x)^{i}=\frac{\partial x^{i}}{\partial \xi^{j}} v(\xi)^{j}, \quad \tilde{\sigma}(x)_{i}=\frac{\partial \xi^{j}}{\partial x^{i}} \sigma(\xi)_{j} .
$$


We also need the pull back of the metric, which is a twice covariant tensor $g=g_{i j} \mathrm{~d} x^{i} \otimes \mathrm{d} x^{j}$. This is given by

$$
\tilde{g}(x)_{i j}=\left[\left(\xi^{*} g\right)(x)\right]_{i j}=\frac{\partial \xi^{k}}{\partial x^{i}} \frac{\partial \xi^{l}}{\partial x^{j}} g_{k l}(\xi) .
$$

Correspondingly, $\tilde{\varsigma}$ defined by $\tilde{\varsigma}(x)^{-2}=\operatorname{det} \tilde{g}(x)$ is given by

$$
\tilde{\varsigma}(x)^{-1}=\varsigma^{-1}(\xi) \operatorname{det}\left(\frac{\partial \xi^{i}}{\partial x^{j}}\right),
$$

Substituting these into (C.2) and using $\tilde{\mu}^{i j k}=\tilde{\varsigma} \varepsilon^{i j k}$ gives an explicit formula for the $\alpha$ tensor in terms of derivatives of the map $\xi$. Note that we are not assuming incompressible flow here; if we do then $\tilde{\varsigma}=\varsigma$ and (C.9) becomes the condition that the map $\xi$ must satisfy to be volume preserving (assuming the actual flow and background flow are incompressible also).

To relate this to formulae in the literature, suppose that we are in Euclidean space with the underlying metric $g=\mathrm{d} x^{2}+\mathrm{d} y^{2}+\mathrm{d} z^{2}$ and so $\mu=\mathrm{d} x \wedge \mathrm{d} y \wedge \mathrm{d} z, \varsigma=1$, and allow the maps $\xi$ to be compressible. Then the $\alpha$-effect formula (C.2) becomes

$$
\alpha_{i}^{n p}=-\eta\left\langle\frac{\partial \xi^{q}}{\partial x^{i}} \frac{\partial \xi^{q}}{\partial x^{j}} \tilde{\varsigma} \varepsilon^{j k l} \frac{\partial}{\partial x^{k}}\left(\frac{\partial \xi^{r}}{\partial x^{l}} \frac{\partial \xi^{r}}{\partial x^{m}} \tilde{\varsigma}\right) \frac{1}{2} \varepsilon^{m n p}\right\rangle
$$

with

$$
\tilde{\varsigma}(x)=\operatorname{det}\left(\frac{\partial x^{i}}{\partial \xi^{j}}\right) .
$$

In (C.10) the term $\partial \xi^{r} / \partial x^{l}$ can come out of the differentation with respect to $x^{k}$ and from (C.11) it follows that

$$
\varepsilon^{j k l} \frac{\partial \xi^{q}}{\partial x^{j}} \frac{\partial \xi^{r}}{\partial x^{l}}=\tilde{\varsigma}^{-1} \varepsilon^{q s r} \frac{\partial x^{k}}{\partial \xi^{s}}
$$

so that (C.10) becomes

$$
\alpha_{i}^{n p}=-\eta\left\langle\frac{\partial \xi^{q}}{\partial x^{i}} \varepsilon^{q s r} \frac{\partial}{\partial \xi^{s}}\left(\frac{\partial \xi^{r}}{\partial x^{m}} \tilde{\varsigma} \frac{1}{2} \varepsilon^{m n p}\right)\right\rangle .
$$

Both equations (C.10) and (C.13) are given in Roberts and Soward (2006a $\mathrm{b}$ ) (with $\tilde{\varsigma} \rightarrow \mathcal{J}^{-1}, \xi^{i} \rightarrow x_{i}^{*}$, $x^{i} \rightarrow x_{i}$, to map our notation to theirs).

While the formulae (C.10) and (C.13) may be derived without reference to the framework we present, by following standard rules of multivariate calculus, they simply correspond to applying pull backs and push forwards at different points of the same calculation. We now illustrate this, noting that notation here becomes awkward. Let us temporarily write $g$ as an operator to give $g b=b_{b}$, and $\mu$ as an operator with $\mu B=b$ for $b\lrcorner \mu=B$, and likewise for $\tilde{g}$ and $\tilde{\mu}$. Then we can expand the diffusion operator $-\nabla^{2}$ as

$$
\mathrm{d} \star \mathrm{d} \star B=\mathrm{d} g \mu \mathrm{d} g \mu B .
$$

We set $\tilde{B}=\xi^{*} B, B=\xi_{*} \tilde{B}$, and apply $\xi^{*}$ to (C.14); The term we then have in our pulled back induction equation can be written as

$$
\begin{aligned}
\xi^{*}(\mathrm{~d} g \mu \mathrm{d} g \mu B) & =\xi^{*}\left(\mathrm{~d} g \mu \mathrm{d} g \mu \xi_{*} \tilde{B}\right) \\
& =\mathrm{d} \tilde{g} \tilde{\mu} \mathrm{d} \tilde{g} \tilde{\mu} \tilde{B}
\end{aligned}
$$

corresponding to the structure (C.10) for the $\alpha$-effect, or as

$$
\xi^{*}(\mathrm{~d} g \mu \mathrm{d} g \mu B)=\mathrm{d} \xi^{*}\left[g \mu \mathrm{d} g \xi_{*}(\tilde{\mu} \tilde{B})\right],
$$

in line with (C.13). There is flexibility to undertake operations at different points on $\mathcal{M}$, and this is clarified by the language of pull backs and push forwards, albeit that it is not easy to write out cleanly in this calculation. On the other hand the differential geometric setting does avoid explicit repeated use of the chain rule and properties of determinants; these are built in. 


\section{References}

Alfvén, H. and Falthammar, G., Cosmical electrodynamics, International Series of Monographs on Physics, 1950 (Oxford: Clarendon Press).

Andrews, D.G. and McIntyre, M.E., An exact theory of nonlinear waves on a Lagrangian-mean flow. J. Fluid Mech., 1978a, 89, 609-646.

Andrews, D.G. and McIntyre, M.E., On wave-action and its relatives. J. Fluid Mech., 1978b, 89, 647-664.

Arnold, V.I., Sur la géométrie différentielle des groupes de Lie de dimension infinie et ses applications à l'hydrodynamique des fluides parfaits. Ann. Inst. Fourier, 1966, 16, 316-361.

Arnold, V.I. and Khesin, B.A., Topological methods in hydrodynamics, Applied Mathematical Sciences, vol. 125, 1998 (Springer-Verlag).

Arter, W., Potential vorticity formulation of compressible magnetohyodynamics. Phys. Rev. Lett., 2013a, 110, 015004.

Arter, W., Geometric results for compressible magnetohydrodynamics. Preprint, arXiv: 1309.7172v1, 2013b.

Besse, N. and Frisch, U., Geometric formulation of the Cauchy invariants for incompressible Euler flow in flat and curved spaces. J. Fluid Mech., 2017, 825, 412-478.

Braginsky, S.I., Self excitation of a magnetic field during the motion of a highly conducting fluid. JETP, 1964a, 47, 1084-1098 [Engl. transl. Sov. Phys. JETP, 1965, 20, 726-735)].

Braginsky, S.I., Theory of the hydromagnetic dynamo. JEPTP, 1964b, 47, 2178-2193 [Engl. transl. Sov. Phys. JETP, 1965, 20, 1462-1471].

Bühler, O., Waves and mean flows, 2009 (Cambridge University Press).

D'Avignon, E., Morrison, P.J. and Pegoraro, F., Action principle for relativistic magnetohydrodynamics. Phys. Rev. D, 2015, 91, 084050.

Eckart, C., Some transformations of the hydrodynamic equations. Phys. Fluids, 1963, 6, 1037-1041.

Enciso, A., Peralta-Salas, D. and Torres de Lizaur, F., Helicity is the only integral invariant of volumepreserving transformations. Proc. Natl. Acad. Sci. U.S.A., 2016, 113, 2035-2040.

Ertel, H., Ein neuer hydrodynamischer Wirbelsatz. (A new hydrodynamic eddy theorem.) Meteorol. Z., 1942, 59, 277-281.

Foias, C., Holm, D.D. and Titi, E.S., The Navier-Stokes-alpha model of fluid turbulence. Physica D, 2001, 152-153, 505-519.

Frankel, T., The geometry of physics, 1997 (Cambridge University Press).

Frieman, E. and Rotenberg, M., On hydromagnetic stability of stationary equilibria. Rev. Mod. Phys., 1960, 32, 898-902.

Fukumoto, Y., A unified view of topological invariants of fluid flows. Topologica, 2008, 1, 003.

Gilbert, A.D., Riedinger, X.R. and Thuburn, J., On the form of the viscous term for two dimensional Navier-Stokes flows. Q. J. Mech. Appl. Math., 2014, 67, 205-228.

Gilbert, A.D. and Vanneste, J., Geometric generalised Lagrangian-mean theories, J. Fluid Mech., 2018, 839, 95-134. 
Gilbert, A.D. and Vanneste, J., A geometric look at momentum flux and stress in fluid mechanics, 2020a, in preparation.

Gilbert, A.D. and Vanneste, J., A geometric look at thickness-weighted averaging. 2020b, in preparation.

Hawking, S.W. and Ellis, G.F.R., The large scale structure of space-time, 1973 (Cambridge University Press).

Holm, D.D., Variational principles for Lagrangian-averaged fluid dynamics. J. Phys. A. Math. Gen., 2020a, 35, 679-668.

Holm, D.D., Lagrangian averages, averaged Lagrangians, and the mean effects of fluctuations in fluid dynamics. Chaos, 2002b, 12 518-530.

Holm, D.D., Marsden, J.E., Ratiu, T. and Weinstein, A., Nonlinear stability of fluid and plasma equilibria. Phys. Rep., 1985, 123, 1-116.

Holm, D.D., Marsden, J.E. and Ratiu, T., The Euler-Poincaré equations and semi-direct products with applications to continuum theories. Adv. Math., 1998, 137, 1-81.

Kawazura, Y., Yoshida, Z. and Fukumoto, Y., Relabeling symmetry in relativistic fluids and plasmas. J. Phys. A: Math. Theor., 2014, 47, 465501.

Kraus, M., Tassic, E. and Grassod, D., Variational integrators for reduced magnetohydrodynamics. J. Comp. Phys., 2016, 321, 435-458.

Leok, M. and Shingel, T., General techniques for constructing variational integrators. Front. Math. China, $2012,7,273-303$.

Lingam, M., Miloshevich, G. and Morrison, P.J., Concomitant Hamiltonian and topological structures of extended magnetohydrodynamics. Phys. Lett. A, 2016, 380, 2400-2406.

Machon, T. The Godbillon-Vey Invariant as a restricted Casimir of three-dimension ideal fluids. J. Phys. A, 2020, 53, 235701 .

Marsden, J.E. and Shkoller, S., Global well-posedness for the Lagrangian averaged Navier-Stokes (LANS- $\alpha$ ) equations on bounded domains. Phil. Trans. R. Soc Lond., 2001, 359, 1449-1468.

McIntyre, M.E. and Shepherd, T.G., An exact local conservation theorem for finite-amplitude disturbances to non-parallel shear flows, with remarks on Hamiltonian structure and on Arnol'd's stability theorems. J. Fluid Mech., 1987, 181, 527-565.

Moffatt, H.K. and Dormy, E. Self-exciting fluid dynamos, 2019 (Cambridge University Press).

Morrison, P.J., Poisson brackets for fluids and plasmas. AIP Conf. Proc., 1982, 88, 13-46.

Morrison, P.J., Hamiltonian description of the ideal fluid. Rev. Mod. Phys., 1998, 70, 467-521.

Morrison, P.J., Andreussi, T. and Pegoraro, F., Lagrangian and Dirac constraints for the ideal incompressible fluid and magnetohydrodynamics. J. Plasma Phys., 2020, 86, 835860301.

Newcomb, W.A., Lagrangian and Hamiltonian methods in magnetohydrodynamics. Nucl. Fusion Suppl, 1962 , part 2, 451-463.

Padhiye, N. and Morrison, P.J., Fluid element relabelling symmetry. Phys. Lett. A, 1996, 219, $287-292$.

Palha, A. and Gerritsma, M., A mass, energy, enstrophy and vorticity conserving (MEEVC) mimetic spectral element discretization for the 2D incompressible Navier-Stokes equations. J. Comp. Phys., 2017, 328, 200220 . 
Qin, H., Guan, X. and Tang, W.M., Variational symplectic algorithm for guiding center dynamics and its application in tokamak geometry. Phys. Plasmas, 2009, 16, 042510.

Roberts, P.H. and Soward, A.M., Eulerian-Lagrangian means in rotating magnetohydrodynamic flows I. General results. Geophys. Astrophys. Fluid Dynam., 2006a, 100, 457-483.

Roberts, P.H. and Soward, A.M., Covariant description of non-relativistic magnetohydrodynamics. Geophys. Astrophys. Fluid Dynam., 2006b, 100, 485-502.

Salmon, R., Hamiltonian fluid mechanics. Ann. Rev. Fluid Mech., 1988, 20, 225-256.

Schutz, B. Geometrical methods of mathematical physics, 1980 (Cambridge University Press).

Shepherd, T.G., Symmetries, conservation laws, and Hamiltonian structure in geophysical fluid dynamics. Adv. Geophys., 1990, 32, 287-338.

Soward, A.M., A kinematic theory of large magnetic Reynolds number dynamos. Phil. Trans. Roy. Soc. A, 1972, 272, 431-462.

Soward, A.M. and Roberts, P.H., The hybrid Euler-Lagrange procedure using an extension of Moffatt's method. J. Fluid Mech., 2010, 661, 45-72.

Soward, A.M. and Roberts, P.H., Eulerian and Lagrangian means in rotating, magnetohydrodynamic flows II. Braginsky's nearly axisymmetric dynamo. Geophys. Astrophys. Fluid Dynam., 2014, 108, 269-322.

Stein, L. Notes on the pull-back connection. Available online at https://duetosymmetry.com/notes/noteson-the-pullback-connection/ (accessed 12th October 2020).

Thuburn, J. and Cotter, C.J., A primal-dual mimetic finite element scheme for the rotating shallow water equations on polygonal spherical meshes J. Comp. Phys., 2015, 290, 274-297.

Trautman, A., Deformations of the Hodge map and optical geometry. J. Geom. E Phys., 1984, 1, 85-95.

Tur, A. V. and Yanovsky, V. V., Invariants in dissipationless hydrodynamic media. J. Fluid Mech., 1993, 248, 67-106.

Van Kampen, N.G. and Felderhoff, B.U., Theoretical methods in plasma physics, 1967 (North-Holland, Amsterdam; Interscience (Wiley)).

Walén, C., On the theory of sunspots. Arkiv f. Mat., Astron. o. Fys., 1944, 30A, 1-87.

Webb, G., Magnetohydrodynamics and fluid dynamics: action principles and conservation laws, Lecture Notes in Physics, vol. 946, 1944 (Springer-Verlag).

Webb, G., Dasgupta, B., McKenzie, J.F., Hu, Q. and Zank, G.P., Local and nonlocal advected invariants and helicities in magnetohydrodynamics and gas dynamics I: Lie dragging approach. J. Phys. A Math. Theor., 2014a, 47, 095501.

Webb, G., Dasgupta, B., McKenzie, J.F., Hu, Q. and Zank, G.P., Local and nonlocal advected invariants and helicities in magnetohydrodynamics and gas dynamics: II. Noether's theorems and Casimirs. J. Phys. A Math. Theor., 2014b, 47, 095502.

Webb,. G.M., Prasad, A., Anco, S.C. and Hu, Q., Godbillon-Vey helicity and magnetic helicity in magnetohydrodynamics. J. Plasma Phys., 2019, 85, 775850502.

Yoshida, K., Kawazura, Y. and Yokoyama, T., Relativistic helicity and link in Minkowski space-time. $J$. Math. Phys. , 2014, 55, 043101. 